\title{
BLOW-UP ANALYSIS FOR NODAL RADIAL SOLUTIONS IN MOSER-TRUDINGER CRITICAL EQUATIONS IN $\mathbb{R}^{2}$
}

\author{
MASSIMO GROSSI ${ }^{\dagger}$ AND DAISUKE NAIMEN ${ }^{\ddagger}$
}

ABSTRACT. In this paper we consider nodal radial solutions $u_{\varepsilon}$ to the problem

$$
\begin{cases}-\Delta u=\lambda u e^{u^{2}+|u|^{1+\varepsilon}} & \text { in } B, \\ u=0 & \text { on } \partial B .\end{cases}
$$

and we study their asymptotic behaviour as $\varepsilon \searrow 0$.

We show that when $u_{\varepsilon}$ has $k$ interior zeros, it exhibits a multiple blowup behaviour in the first $k$ nodal sets while it converges to the least energy solution of the problem with $\varepsilon=0$ in the $(k+1)$-th one. We also prove that in each concentration set, with an appropriate scaling, $u_{\varepsilon}$ converges to the solution of the classical Liouville problem in $\mathbb{R}^{2}$.

Keywords: Radial solutions, Moser-Trudinger inequality.

AMS Subject Classifications: 35B32, 35J61.

\section{INTRODUCTION}

In this paper we study the asymptotic behavior of nodal radial solutions to

$$
\begin{cases}-\Delta u=f_{\varepsilon}(u) & \text { in } B \\ u=0 & \text { on } \partial B,\end{cases}
$$

where $f_{\varepsilon}(s)$ is some smooth nonlinearity of Moser-Trudinger type depending on a positive parameter $\varepsilon$ goings to zero. In what follows we will be more precise on the conditions on $f_{\varepsilon}(s)$. In (1.1) $B$ is the unit ball of $\mathbb{R}^{2}$.

First let us recall some classical results when $u$ is a positive solution of (1.1). In this case our problem is linked to the celebrated Moser-PohozaevTrudinger inequality ([20], 23], 26], ) namely

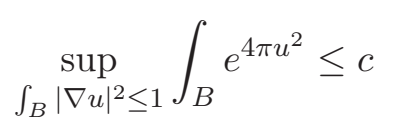

for any $u$ in the Sobolev space $W_{0}^{1,2}(B)$.

In the pioneering paper [11] it was proved that the supremum in (1.2) is achieved and the corresponding Eulero-Lagrange equation is given by

$$
\begin{cases}-\Delta u=\frac{u e^{u^{2}}}{\int_{B} u^{2} e^{u^{2}}} & \text { in } B \\ u=0 & \text { on } \partial B\end{cases}
$$

The first author is supported by PRIN-2015 grant (P.I. A. Malchiodi).

The second author was supported by Grant-in-Aid for JSPS Research Fellow Grant Number JP15J12092. 
Now we consider the related (but not equivalent) problem

$$
\begin{cases}-\Delta u=\lambda u e^{u^{2}} & \text { in } B \\ u=0 & \text { on } \partial B .\end{cases}
$$

In 22 there is an interesting discussion on relationship between (1.3) and (1.4).

In [1] it was proved the existence of solutions to (1.4) for any $\lambda \in\left(0, \lambda_{1}\right)$ where $\lambda_{1}$ is the first eigenvalue of $-\Delta$ with Dirichlet boundary conditions (see also [13]). As $\lambda \rightarrow 0$, the corresponding solution $u_{\lambda}$ concentrates around the origin and its asymptotic behavior was studied in 22 and 3 .

These results hold also for more general problems like

$$
\begin{cases}-\Delta u=\lambda f(u) e^{u^{2}} & \text { in } B \\ u=0 & \text { on } \partial B .\end{cases}
$$

We refer to [1] and [13] for the precise assumptions on $f$.

If we consider the case of sign changing radial solutions we find some interesting differences. Indeed, in [5] the authors showed that in order to have existence results of nodal solutions we need to impose some restrictions on the nonlinearity $f$ in (1.5). A particular case is the following,

Theorem 1.1. (See [5] and [6]) Let us consider the problem

$$
\begin{cases}-\Delta u=\lambda u e^{u^{2}+|u|^{\beta}} & \text { in } B \\ u=0 & \text { on } \partial B .\end{cases}
$$

Then we have that,

i) if $1<\beta<2$ there exists a radial solution with $k$ interior zeros for any integer $k \geq 1$ and for any $\lambda \in\left(0, \lambda_{1}\right)$,

ii) if $0 \leq \beta \leq 1$ there exists $\lambda=\lambda_{A Y}>0$ such that for any $0<\lambda<\lambda_{A Y}$ there exist no solution to (1.6).

From this result we get that the nonlinearity $f(s)=s e^{s^{2}+s}$ is the border line case between the existence and nonexistence of nodal solutions. Hence it becomes interesting to study the asymptotic behavior of the solution $u_{\varepsilon}$ in (1.6) as $\beta=1+\varepsilon, 0<\lambda<\lambda_{A Y}$ and $\varepsilon \searrow 0$.

In order to state our main result we need to introduce some notations. First let us denote by $u_{0}$ the solution of

$$
\begin{cases}-\Delta u=\lambda u e^{u^{2}+u} & \text { in } B, \\ u>0 & \text { in } B, \\ u=0 & \text { on } \partial B .\end{cases}
$$

Next, for $u \in H_{0}^{1}(B)$ and $\varepsilon \geq 0$ let us consider the functional

$$
I_{\varepsilon}(u)=\frac{1}{2} \int_{B}|\nabla u|^{2}-\int_{B} F_{\varepsilon}(u)
$$

where $F_{\varepsilon}(s)=\lambda \int_{0}^{s} t e^{t^{2}+|t|^{1+\varepsilon}} d t$. We have the following result, 
Theorem 1.2 (Global behavior). Let $u_{\varepsilon}$ be a nodal radial solution obtained in [6] which verifies

$$
\begin{cases}-\Delta u=\lambda u e^{u^{2}+|u|^{1+\varepsilon}} & \text { in } B, \\ u=0 & \text { on } \partial B .\end{cases}
$$

with $k$ interior zeros denoted by $0=r_{0, \varepsilon}<r_{1, \varepsilon}<r_{2, \varepsilon}<\cdots<r_{k, \varepsilon}<$ $1=r_{k+1, \varepsilon}$. Assume that $u_{\varepsilon}(0)>0$. Then we have that, as $\varepsilon \rightarrow 0$ and $0<\lambda<\lambda_{A Y}$,

$$
\begin{gathered}
u_{\varepsilon}(x) \rightarrow(-1)^{k} u_{0}(x) \quad \text { in } C_{l o c}^{2}(B \backslash\{0\}) \\
r_{i, \varepsilon} \rightarrow 0 \quad \text { for any } i=1, \ldots, k, \\
\int_{B}\left|\nabla u_{\varepsilon}\right|^{2} \rightarrow \int_{B}\left|\nabla u_{0}\right|^{2}+4 k \pi, \\
I_{\varepsilon}\left(u_{\varepsilon}\right) \rightarrow I_{0}\left(u_{0}\right)+2 k \pi .
\end{gathered}
$$

Theorem 1.3 (Local behavior). Let $u_{\varepsilon}$ be the solution considered in the previous theorem. Then for $i=0, \ldots, k$ let $M_{i, \varepsilon} \in\left(r_{i-1, \varepsilon}, r_{i, \varepsilon}\right)=A_{i, \varepsilon}$ be the points such that $u_{\varepsilon}\left(M_{i, \varepsilon}\right)=\left\|u_{\varepsilon}\right\|_{L^{\infty}\left(A_{i, \varepsilon}\right)}$ (we have that $\left.M_{1, \varepsilon}=0\right)$. Then if $\delta_{i, \varepsilon}$ is defined as $\delta_{i, \varepsilon}=r_{i, \varepsilon} \gamma_{i, \varepsilon}$ with

$$
2 \lambda r_{i, \varepsilon}^{2} e^{\left\|u_{\varepsilon}\right\|_{L^{\infty}\left(A_{i, \varepsilon}\right)}^{2}+\left\|u_{\varepsilon}\right\|_{L^{\infty}\left(A_{i, \varepsilon}\right)}^{1+\varepsilon}}\left\|u_{\varepsilon}\right\|_{L^{\infty}\left(A_{i, \varepsilon}\right)} \gamma_{i, \varepsilon}^{2}=1
$$

we have that $\delta_{i, \varepsilon} \rightarrow 0$ and

$$
\begin{aligned}
2\left\|u_{\varepsilon}\right\|_{L^{\infty}\left(r_{i-1, \varepsilon}, r_{i, \varepsilon}\right)}\left(u_{\varepsilon}\left(M_{i, \varepsilon}+\delta_{i, \varepsilon} r\right)\right. & \left.-\left\|u_{\varepsilon}\right\|_{L^{\infty}\left(r_{i-1, \varepsilon}, r_{i, \varepsilon}\right)}\right) \\
& \rightarrow \log \frac{1}{\left(1+\frac{r^{2}}{8}\right)^{2}} \quad \text { in } C_{l o c}^{1}(0,+\infty) .
\end{aligned}
$$

Remark 1.4. Another interesting problem with similar behavior is given by

$$
\begin{cases}-\Delta u=\lambda u e^{u^{2-\varepsilon}} & \text { in } B, \\ u=0 & \text { on } \partial B .\end{cases}
$$

As for (1.9) it is possible to show that there exists a family of nodal solutions $u_{\varepsilon}$ for any $\varepsilon>0$. Despite the nonlinearity is not covered by the assumptions of Theorem 1.3 in [6] we can still repeat the proof in order to get the existence result. Moreover the result in [5] applies and so there exists a constant $\bar{\lambda}$ such that for any $\lambda \in(0, \bar{\lambda})$ there exists no sign changing solution.

It is possible to show that analogous results like in Theorems 1.2 and 1.3 hold. The interest in this type of nonlinearity is given by the similarity with the analogous in higher dimension (see problem (1.22) and the comments below).

Remark 1.5. Similar phenomena to Theorem 1.1, 1.2 and 1.3 appears in higher dimensions for the problem

$$
\begin{cases}-\Delta u=|u|^{\frac{4}{N-2}} u+\lambda u & \text { in } B, \\ u=0 & \text { on } \partial B .\end{cases}
$$


where $N \geq 3$ and $B$ is the unit ball of $\mathbb{R}^{N}$.

In [8] it was proved that if $N=4,5,6$ there exists $\lambda^{*}>0$ such that there is no nodal radial solution for $0<\lambda<\lambda^{*}$. The asymptotic behavior of the solution $u_{\lambda}$ as $\lambda \rightarrow \bar{\lambda}$ for a limit value $\bar{\lambda}>0$ and $N=4,5,6$ was studied in [17]. Note that the case $N=6$ has strong similarities with our results for the case $k=1$. Other existence results for $N=4,5$ can be founded in [18].

It is interesting to compare the previous results with other similar problems like

$$
\left\{\begin{array}{l}
-\Delta u=|u|^{p-1} u \text { in } B \subset \mathbb{R}^{2} \\
u=0 \text { on } \partial B
\end{array}\right.
$$

(see [15]) and

$$
\left\{\begin{array}{l}
-\Delta u=\lambda \sinh \text { in } B \subset \mathbb{R}^{2} \\
u=0 \text { on } \partial B
\end{array}\right.
$$

(see [16]).

Both this problems share the feature that suitable transformations of positive solutions converge to the limit problem

$$
\left\{\begin{array}{l}
-\Delta u=e^{u} \text { in } \mathbb{R}^{2} \\
\int_{\mathbb{R}^{2}} e^{u}<+\infty
\end{array}\right.
$$

We want to compare Theorems 1.2 and 1.3 with the analogous ones for 1.18 and 1.19 ,

The global behavior is different: indeed solutions founded in [16] converge to suitable multiple of the Green function which does not belong to $W_{0}^{1.2}(B)$ and solutions studied in [15] goes to 0 everywhere.

However more striking differences appear if we look at the local behavior. Indeed, in this case the solutions to (1.18) and (1.19) involve the singular Liouville problem

$$
\left\{\begin{array}{l}
-\Delta u=|x|^{\alpha} e^{u} \text { in } \mathbb{R}^{2} \\
\int_{\mathbb{R}^{2}}|x|^{\alpha} e^{u}<+\infty
\end{array}\right.
$$

for some suitable positive number $\alpha$. We refer to [15] and [16] for more precises statements. In our case the local behavior of the solution is again related to the problem (1.20). In some sense our problem is more similar to the "almost critical" problem in higher dimensions $N \geq 3$ given by

$$
\left\{\begin{array}{l}
-\Delta u=|u|^{\frac{4}{N-2}-\varepsilon} u \text { in } B \subset \mathbb{R}^{N} \\
u=0 \text { on } \partial B .
\end{array}\right.
$$

In this case the local behavior of nodal solutions is given by the (unique) positive smooth solution of the limit problem (see [10], [12] and [24])

$$
-\Delta u=u^{\frac{N+2}{N-2}} \text { in } \mathbb{R}^{N} \text {. }
$$

In our opinion this similarity is due to the effect of nonlinearity which is very close to those in Moser-Trudinger inequality.

The paper is organized as follows: in Section 2 we prove some energy estimates for the solution $u_{\varepsilon}$. In Section 3 we study the behavior of $u_{\varepsilon}$ in the 
ball $B_{r_{1, \varepsilon}}$ where $r_{1, \varepsilon}$ is the first zero of $u_{\varepsilon}$. In Section 4 and 5 we consider the behavior of $u_{\varepsilon}$ in the other annular regions and in Section 6 we give the proof of Theorems 1.2 and 1.3 . Finally in Appendix $\mathrm{A}$ we prove some technical lemmas.

For all $u \in H_{0}^{1}(B)$, we define $\|u\|:=\left(\int_{B}|\nabla u|^{2} d x\right)^{1 / 2}$. In addition, let $B(0, r):=B_{r}$ and $B(r, s):=B_{s} \backslash B_{r}$ for $r, s>0$.

\section{Energy estimates For $u_{\varepsilon}$}

In the following, we always assume $0<\lambda<\min \left\{\lambda_{1}, \lambda_{\mathrm{AY}}\right\}$ and we consider the least energy nodal solution $u_{\varepsilon}$ of (1.9) obtained by Theorem 1.3 in [6]. More precisely, we define $H_{\mathrm{r}, 0}^{1}(B)$ as a subspace of $H_{0}^{1}(B)$ which consists of all the radial functions and by the Nehari manifold

$$
\mathcal{N}_{\varepsilon}=\left\{\left.u \in H_{\mathrm{r}, 0}^{1}(B) \backslash\{0\}\left|\int_{B}\right| \nabla u\right|^{2} d x=\int_{B} f_{\varepsilon}(u) u d x\right\},
$$

and for $k \in \mathbb{N}$,

$$
\begin{aligned}
\mathcal{N}_{k, \varepsilon}:= & \left\{u \in H_{\mathrm{r}, 0}^{1}(B) \mid \exists r_{i} \in(0,1) ; 0=r_{0}<r_{1}<\cdots<r_{k+1}=1,\right. \\
& u\left(r_{i}\right)=0, u_{i}:=\left.u\right|_{\left.B_{\left(r_{i-1}, r_{i}\right)},(-1)^{i-1} u_{i}>0, u_{i} \in \mathcal{N}_{\varepsilon}, 1 \leq i \leq k+1\right\} .}
\end{aligned}
$$

Then let $u_{\varepsilon} \in \mathcal{N}_{k, \varepsilon}$ be a solution to (1.9) such that

$$
I_{\varepsilon}\left(u_{\varepsilon}\right)=\inf _{u \in \mathcal{N}_{k, \varepsilon}} I_{\varepsilon}(u) .
$$

We choose constants $0=r_{0, \varepsilon}<r_{1, \varepsilon}<\cdots<r_{k, \varepsilon}<r_{k+1, \varepsilon}=1$ so that $u_{\varepsilon}\left(r_{i, \varepsilon}\right)=0$ for $i=1,2, \cdots, k$. Moreover, for each $i=1,2, \cdots, k+1$, define $u_{i, \varepsilon}:=\left.u_{\varepsilon}\right|_{B\left(r_{i-1, \varepsilon}, r_{i}\right)}$ with zero extension to whole $B$.

First let us show a suitable upper bound for $I_{\varepsilon}\left(u_{\varepsilon}\right)$. To this end, we use the Moser function defined in [2]. For $0<l<R \leq 1$, we define

$$
m_{l, R}(x):=\frac{1}{\sqrt{2 \pi}} \begin{cases}\left(\log \frac{R}{l}\right)^{\frac{1}{2}} & 0 \leq|x|<l \\ \frac{\log \frac{R}{|x|}}{\left(\log \frac{R}{l}\right)^{\frac{1}{2}}} & l \leq|x| \leq R \\ 0 & |x|>R .\end{cases}
$$

Then it satisfies $m_{l, R} \in H_{0}^{1}(B)$ and $\left\|m_{l, R}\right\|=1$. In addition let us define a cut off function,

$$
\phi_{l, R}(x)=1-\frac{m_{l, R}(x)}{\sqrt{2 \pi}^{-1}\left(\log \frac{R}{l}\right)^{\frac{1}{2}}} \in H^{1}(B)
$$

Then we have $0 \leq \phi_{l, R} \leq 1, \phi_{l, R}=0$ on $B_{l}$ and $\phi_{l, R}=1$ on $B \backslash B_{R}$. For $0<l_{1}=l_{1, \varepsilon}<R_{1}=R_{1, \varepsilon}<p_{1}=p_{1, \varepsilon}<l_{2}=l_{2, \varepsilon}<R_{2}=R_{2, \varepsilon}<p_{2}=p_{2, \varepsilon}<$ $\cdots<l_{k}=l_{k, \varepsilon}<R_{k}=R_{k, \varepsilon}<1$, we set

$$
\left\{\begin{array}{l}
w_{1, \varepsilon}:=m_{l_{1}, R_{1}}, \\
w_{i, \varepsilon}:=(-1)^{i-1} \phi_{R_{i-1}, p_{i-1}} m_{l_{i}, R_{i}} \text { for } k=2, \cdots, k, \text { and } \\
w_{k+1, \varepsilon}:=(-1)^{k} \phi_{R_{k}, 1} u_{0},
\end{array}\right.
$$


where $u_{0}$ is the least energy solution of (1.7) obtained in [2] and thus, it satisfies

$$
I_{0}\left(u_{0}\right)=\inf _{u \in \mathcal{N}_{0}} I_{0}(u) \in(0,2 \pi) .
$$

We choose $l_{1}, R_{1}, p_{1}, \cdots, l_{k}, R_{k}$ so that $R_{k} \rightarrow 0$ and

$$
\left\{\begin{array}{l}
\frac{\log \frac{1}{R_{i}}}{\log \frac{1}{l_{i}}} \rightarrow 0(i=1,2, \cdots, k), \\
\frac{\log \frac{R_{i}}{l_{i}}}{\log \frac{p_{i-1}}{R_{i-1}}} \rightarrow 0, \quad \frac{p_{i-1}}{l_{i}} \rightarrow 0(i=2, \cdots, k),
\end{array}\right.
$$

as $\varepsilon \rightarrow 0$. For example, take any $R_{k}>0$ such that $R_{k} \rightarrow 0$ as $\varepsilon \rightarrow 0$ and then, choose $l_{k}=e^{-1 / R_{k}}, p_{k-1}=l_{k}^{2}$, and $R_{k-1}=p_{k-1} e^{-1 / l_{k}}$. Similarly, set $l_{k-1}=e^{-1 / R_{k-1}}, p_{k-2}=l_{k-1}^{2}, R_{k-2}=p_{k-2} e^{-1 / l_{k-1}}$ and so on. We note that, for every $i=1,2, \cdots, k+1$ and $\varepsilon \in(0,1)$, there exists a constant $t_{i, \varepsilon}>0$ such that $t_{i, \varepsilon} w_{i, \varepsilon} \in \mathcal{N}_{\varepsilon}$. (See Step 2 in the proof of Lemma 3.4 in [2].) We define a test function

$$
w_{\varepsilon}(x):=\sum_{i=1}^{k+1} t_{i, \varepsilon} w_{i, \varepsilon} .
$$

Then we have $w_{\varepsilon} \in \mathcal{N}_{k, \varepsilon}$. We obtain the following.

Lemma 2.1. We get

$$
\limsup _{\varepsilon \rightarrow 0} I_{\varepsilon}\left(u_{\varepsilon}\right) \leq 2 \pi k+I_{0}\left(u_{0}\right) .
$$

Proof. First observe that since $w_{\varepsilon} \in \mathcal{N}_{k, \varepsilon}$, we have

$$
I\left(u_{\varepsilon}\right) \leq I_{\varepsilon}\left(w_{\varepsilon}\right)=\sum_{i=1}^{k+1} I_{\varepsilon}\left(t_{i, \varepsilon} w_{i, \varepsilon}\right) .
$$

Then it suffices to show,

(I) $\limsup _{\varepsilon \rightarrow 0} I_{\varepsilon}\left(t_{1, \varepsilon} w_{1, \varepsilon}\right) \leq 2 \pi$,

(II) $\lim \sup _{\varepsilon \rightarrow 0} I_{\varepsilon}\left(t_{i, \varepsilon} w_{i, \varepsilon}\right) \leq 2 \pi$, for $i=2, \cdots, k$, and,

(III) $\lim \sup _{\varepsilon \rightarrow 0} I_{\varepsilon}\left(t_{k+1, \varepsilon} w_{k+1, \varepsilon}\right) \leq I_{0}\left(u_{0}\right)$.

(I) We claim

$$
\limsup _{\varepsilon \rightarrow 0} t_{1, \varepsilon}^{2} \leq 4 \pi
$$

If not, there exist a sequence $\left(\varepsilon_{n}\right)$ and a constant $\delta>0$ such that $\varepsilon_{n} \rightarrow 0$ as $n \rightarrow \infty$ and $t_{1, \varepsilon_{n}}^{2} \geq 4 \pi(1+\delta)$ for all $n$. Set $t_{n}:=t_{1, \varepsilon_{n}}, w_{n}:=w_{1, \varepsilon_{n}}$, $l_{n}:=l_{1, \varepsilon_{n}}$ and $R_{n}:=R_{1, \varepsilon_{n}}$ for simplicity. Since $t_{n} w_{n} \in \mathcal{N}_{\varepsilon_{n}}$, we get

$$
t_{n}^{2}\left\|w_{n}\right\|^{2}=\lambda \int_{B}\left(t_{n} w_{n}\right)^{2} e^{\left|t_{n} w_{n}\right|^{2}+\left|t_{n} w_{n}\right|^{1+\varepsilon_{n}}} d x .
$$

Then we have

$$
\begin{aligned}
t_{n}^{2} & \geq \lambda \int_{B_{l_{n}}}\left(t_{n} w_{n}\right)^{2} e^{\left|t_{n} w_{n}\right|^{2}+\left|t_{n} w_{n}\right|^{1+\varepsilon_{n}}} d x \\
& \geq \frac{\lambda}{2} t_{n}^{2} l_{n}^{2} \log \frac{R_{n}}{l_{n}} e^{\frac{t_{n}^{2}}{2 \pi} \log \frac{R_{n}}{l_{n}}} \\
& =\frac{1}{2} t_{n}^{2} \log \frac{R_{n}}{l_{n}} \exp \left\{\frac{t_{n}^{2}}{2 \pi}\left(\log \frac{1}{l_{n}}-\log \frac{1}{R_{n}}\right)-2 \log \frac{1}{l_{n}}\right\} .
\end{aligned}
$$


Here, (2.1) implies that

$$
\log \frac{1}{R_{n}}=o\left(\log \frac{1}{l_{n}}\right)
$$

It follows that

$$
\log \frac{R_{n}}{l_{n}} \rightarrow \infty(n \rightarrow \infty) .
$$

As a consequence, we find a constant $\delta^{\prime}>0$ such that

$$
2 \geq \exp \left\{\delta^{\prime} \log \frac{1}{l_{n}}\right\}
$$

for large $n$. Taking $n \rightarrow \infty$, we have a contradiction. Now, since $t_{1, \varepsilon} w_{1, \varepsilon} \in$ $\mathcal{N}_{\varepsilon},\left\|w_{1, \varepsilon}\right\|=1$ and $\lim \sup _{\varepsilon \rightarrow 0} t_{1, \varepsilon}^{2} \leq 4 \pi$, we get

$$
\left|\int_{B} f_{\varepsilon}\left(t_{1, \varepsilon} w_{1, \varepsilon}\right) t_{1, \varepsilon} w_{1, \varepsilon} d x\right| \leq C
$$

for some constant $C>0$ uniformly for $\varepsilon>0$. Furthermore, note $t_{1, \varepsilon} w_{1, \varepsilon} \rightarrow 0$ a.e. on $B$. Then by Lemma A.1 in Appendix $\mathrm{A}$, we find

$$
\lim _{\varepsilon \rightarrow 0} \int_{B} F_{\varepsilon}\left(t_{1, \varepsilon} w_{1, \varepsilon}\right) d x=\int_{B} F_{0}(0) d x=0 .
$$

As a consequence, we get

$$
\limsup _{\varepsilon \rightarrow 0} I_{\varepsilon}\left(t_{1, \varepsilon} w_{1, \varepsilon}\right)=\limsup _{\varepsilon \rightarrow 0} \frac{t_{1, \varepsilon}^{2}}{2} \leq 2 \pi .
$$

This finishes the proof of (I).

(II) Fix $i=2,3, \cdots, k$. We first claim $\lim _{\varepsilon \rightarrow 0} \int_{B}\left|\nabla w_{i, \varepsilon}\right|^{2} d x=1$. In fact, we get

$$
\begin{aligned}
\int_{B}\left|\nabla w_{i, \varepsilon}\right|^{2} d x & =\int_{B}\left|\nabla \phi_{R_{i-1}, p_{i-1}}\right|^{2} m_{l_{i}, R_{i}}^{2} d x+2 \int_{B} \phi_{R_{i-1}, p_{i-1}} m_{l_{i}, R_{i}} \nabla \phi_{R_{i-1}, p_{i-1}} \nabla m_{l_{i}, R_{i}} d x \\
& +\int_{B}\left|\nabla m_{l_{i}, R_{i}}\right|^{2} \phi_{R_{i-1}, p_{i-1}}^{2} d x \\
& =I_{1}+I_{2}+I_{3} .
\end{aligned}
$$

It follows from (2.1) that

$$
I_{1}=\int_{B\left(R_{i-1}, p_{i-1}\right)}\left|\nabla \phi_{R_{i-1}, p_{i-1}}\right|^{2} m_{l_{i}, R_{i}}^{2} d x=\frac{\log \frac{R_{i}}{l_{i}}}{\log \frac{p_{i-1}}{R_{i-1}}} \rightarrow 0
$$

as $\varepsilon \rightarrow 0$. Since $\phi_{R_{i-1}, p_{i-1}} m_{l_{i}, R_{i}} \nabla \phi_{R_{i-1}, p_{i-1}} \nabla m_{l_{i}, R_{i}}=0$ on $B$, we get $I_{2}=0$. Furthermore, as $\phi_{R_{i-1}, p_{i-1}}=1$ on $B\left(l_{i}, R_{i}\right)$ and $\nabla m_{l_{i}, R_{i}}=0$ on $B_{l_{i}}$, we clearly have

$$
I_{3}=\int_{B}\left|\nabla m_{l_{i}, R_{i}}\right|^{2} d x=1
$$

This shows the claim. Now we shall show $\lim \sup _{\varepsilon \rightarrow 0} t_{i, \varepsilon}^{2} \leq 4 \pi$. If not, there exists a constant $\delta>0$ such that $t_{i, \varepsilon}^{2} \geq 4 \pi(1+\delta)$ for all small $\varepsilon>0$ by 
extracting a sequence if necessary. Then noting $t_{i, \varepsilon} w_{i, \varepsilon} \in \mathcal{N}_{\varepsilon}$ and (2.1), we get

$$
\begin{aligned}
1+o(1) & =\lambda \int_{B}\left(\phi_{R_{i-1}, p_{i-1}} m_{l_{i}, R_{i}}\right)^{2} \exp \left\{\left(t_{i, \varepsilon} \phi_{R_{i-1}, p_{i-1}} m_{l_{i}, R_{i}}\right)^{2}+\left|t_{i, \varepsilon} \phi_{R_{i-1}, p_{i-1}} m_{l_{i}, R_{i}}\right|^{1+\varepsilon}\right\} d x \\
& \geq \lambda \int_{B\left(p_{i-1}, l_{i}\right)} m_{l_{i}, R_{i}}^{2} \exp \left\{\left(t_{i, \varepsilon} m_{l_{i}, R_{i}}\right)^{2}\right\} d x \\
& =\frac{\lambda}{2} \log \frac{R_{i}}{l_{i}} \exp \left\{\frac{t_{i, \varepsilon}^{2}}{2 \pi}\left(\log \frac{1}{l_{i}}-\log \frac{1}{R_{i}}\right)-2 \log \frac{1}{l_{i}}-2 \log \frac{1}{1-\left(p_{i-1} / l_{i}\right)^{2}}\right\} \\
& \geq C \exp \left(\delta^{\prime} \log \frac{1}{l_{i}}\right),
\end{aligned}
$$

for some constants $C, \delta^{\prime}>0$ if $\varepsilon$ is small enough. Taking $\varepsilon \rightarrow 0$, we get a contradiction. Then, analogously with the conclusion for (I), we obtain

$$
\limsup _{\varepsilon \rightarrow 0} I_{\varepsilon}\left(t_{i, \varepsilon} w_{i, \varepsilon}\right)=\limsup _{\varepsilon \rightarrow 0} \frac{\left\|t_{i, \varepsilon} w_{i, \varepsilon}\right\|^{2}}{2} \leq 2 \pi
$$

This proves (II).

(III) We claim that $t_{k+1, \varepsilon}$ is bounded. To see this, we follow the argument on p493-494 in [6]. We assume on the contrary, for a sequence $\left(\varepsilon_{n}\right)$, we have $\varepsilon_{n} \rightarrow 0$ and $t_{k+1, \varepsilon_{n}} \rightarrow \infty$ as $n \rightarrow \infty$. Then we let

$$
v_{n}:=\frac{t_{k+1, \varepsilon_{n}} w_{k+1, \varepsilon_{n}}}{\left\|t_{k+1, \varepsilon_{n}} w_{k+1, \varepsilon_{n}}\right\|}=\frac{w_{k+1, \varepsilon_{n}}}{\left\|w_{k+1, \varepsilon_{n}}\right\|} .
$$

Then using (2.1), we get $v_{n} \rightarrow v_{0}=u_{0} /\left\|u_{0}\right\| \neq 0$ in $H_{0}^{1}(B)$. Furthermore, noting $t_{i, \varepsilon_{n}}$ is bounded for all $i=1,2, \cdots, k$ as proved in (I) and (II), we obtain

$$
\left\|w_{\varepsilon_{n}}\right\|^{2}=\sum_{i=1}^{k} t_{i, \varepsilon_{n}}^{2}+t_{k+1, \varepsilon_{n}}^{2}\left\|w_{k+1, \varepsilon_{n}}\right\|^{2}=t_{k+1, \varepsilon_{n}}^{2}\left\|w_{k+1, \varepsilon_{n}}\right\|^{2}\left(1+\eta_{n}\right),
$$

for a sequence $\left(\eta_{n}\right) \subset \mathbb{R}^{+}$with $\eta_{n} \rightarrow 0$ as $n \rightarrow \infty$. Therefore, we get

$$
\frac{w_{\varepsilon_{n}}}{\left\|w_{\varepsilon_{n}}\right\|}=\frac{1}{\left(1+\eta_{n}\right)^{\frac{1}{2}}}\left(v_{n}+\sum_{i=1}^{k} \frac{t_{i, \varepsilon_{n}}}{t_{k+1, \varepsilon_{n}}\left\|w_{k+1, \varepsilon_{n}}\right\|} w_{i, \varepsilon_{n}}\right) \rightarrow v_{0} \neq 0 \text { in } H_{0}^{1}(B) .
$$

Finally using $w_{\varepsilon_{n}} \in \mathcal{N}_{\varepsilon_{n}}$ and the Fatou lemma, we have

$$
\begin{aligned}
1 & =\liminf _{n \rightarrow \infty} \frac{1}{\left\|w_{\varepsilon_{n}}\right\|^{2}} \int_{B} f_{\varepsilon_{n}}\left(w_{n}\right) w_{n} d x \\
& \geq \int_{B} \liminf _{n \rightarrow \infty} \frac{f_{\varepsilon_{n}}\left(w_{\varepsilon_{n}}\right)}{w_{\varepsilon_{n}}}\left(\frac{w_{\varepsilon_{n}}}{\left\|w_{\varepsilon_{n}}\right\|}\right)^{2} d x \\
& =\infty,
\end{aligned}
$$

a contradiction. This proves the claim. Finally let us end the proof. We suppose the conclusion of (III) does not hold on the contrary. Then, we have a sequence $\left(\varepsilon_{n}\right)$ and a constant $\delta>0$ such that $\varepsilon_{n} \rightarrow 0$ as $n \rightarrow \infty$ and $I_{\varepsilon_{n}}\left(t_{k+1, \varepsilon_{n}} w_{k+1, \varepsilon_{n}}\right) \geq I_{0}\left(u_{0}\right)+\delta$ for all $n$. On the other hand, as $t_{k+1, \varepsilon_{n}}$ is bounded, there exists a constant $t_{0} \geq 0$ such that $t_{k+1, \varepsilon_{n}} \rightarrow t_{0}$ as $n \rightarrow \infty$ up to subsequences. This implies $t_{k+1, \varepsilon_{n}} w_{k+1, \varepsilon_{n}} \rightarrow t_{0} u_{0}$ in $H_{0}^{1}(\Omega)$ as $n \rightarrow \infty$ 
and then, we get $t_{0} u_{0} \in \mathcal{N}_{0}$. It follows that $t_{0}=0$ or 1 . (See Step 2 in the proof of Lemma 3.4 in [2.) Consequently, we deduce

$$
\lim _{n \rightarrow \infty} I_{\varepsilon_{n}}\left(t_{k+1, \varepsilon_{n}} w_{k+1, \varepsilon_{n}}\right) \leq I_{0}\left(u_{0}\right)
$$

which implies a contradiction. This completes (III).

Lemma 2.2. There exist constants $0<K<K^{\prime}$ such that

$$
K \leq\|u\|^{2} \leq K^{\prime}
$$

for all $u \in \mathcal{N}_{\varepsilon}$ and small $\varepsilon>0$.

Proof. The lower bound is clearly confirmed by Lemma A.2 in Appendix A. On the other hand, the upper bound is proved similarly to claim 1 on p404 in [2]. This finishes the proof.

Next we study the behavior of $r_{i, \varepsilon}$. To this end we recall the next lemma.

Lemma 2.3 (Radial lemma 25]). Let $B^{N} \subset \mathbb{R}$ be a $N$-dimensional unit ball and $H_{\text {rad }}\left(B^{N}\right)$ be a subspace of $H^{1}\left(B^{N}\right)$ which consists of all the radial functions. Then, there exists a constant $c_{N}>0$ such that

$$
|u(r)| \leq c_{N}\|u\| / r^{\frac{N-1}{2}}\left(u \in H_{\text {rad }}\left(B^{N}\right) \text { and } r \in(0,1)\right) .
$$

In particular, for $N=2$ we have $|u(r)| \leq c_{2}\|u\| / \sqrt{r}$.

We deduce the following.

Lemma 2.4. We see

$$
r_{i, \varepsilon} \rightarrow 0 \text { as } \varepsilon \rightarrow 0
$$

for all $i=1,2, \cdots, k$.

Proof. By Lemma 2.2, we may assume $u_{\varepsilon}$ is bounded in $H_{0}^{1}(B)$ and $u_{\varepsilon} \rightarrow u$ weakly in $H_{0}^{1}(B)$ as $\varepsilon \rightarrow 0$ where $u$ is a radial solution $u$ to (1.9) with $\varepsilon=0$. Moreover we recall that $u_{i, \varepsilon}=\left.u_{\varepsilon}\right|_{B\left(r_{i-1, \varepsilon}, r_{i, \varepsilon}\right)}$ satisfies $(-1)^{i-1} u_{i, \varepsilon} \geq 0$ for all $i=1,2, \cdots, k+1$. Then, we can suppose there exists a function $u_{i} \in H_{0}^{1}(B)$ such that $u_{i, \varepsilon} \rightarrow u_{i}$ weakly in $H_{0}^{1}(B)$ and $(-1)^{i-1} u_{i} \geq 0$ for all $i=1,2, \cdots, k+1$ and further, $u=\sum_{i=1}^{k+1} u_{i}$. Now, let us show $r_{k, \varepsilon} \rightarrow 0$ which also implies $r_{i, \varepsilon} \rightarrow 0$ for all $i=1,2, \cdots, k-1$ as $\varepsilon \rightarrow 0$. If not, we may suppose that there exists a constant $r_{k} \in(0,1]$ such that $r_{k, \varepsilon} \rightarrow$ $r_{k}$ as $\varepsilon \rightarrow 0$. We then claim $u_{k+1} \neq 0$. Indeed, if $u_{k+1}=0$, on the contrary, we have $\int_{B} u_{k+1, \varepsilon}^{2} d x \rightarrow 0$ as $\varepsilon \rightarrow 0$. It follows that $\left\|u_{k+1, \varepsilon}\right\|_{\infty}=$ $\sup _{r \in\left(r_{k, \varepsilon}, 1\right)} u_{k+1, \varepsilon}(r) \rightarrow \infty$ as $\varepsilon \rightarrow 0$. Otherwise, from Lemma 2.2, we get

$$
\begin{aligned}
0<K & \leq\left\|u_{k+1, \varepsilon}\right\|^{2}=\lambda \int_{B} u_{k+1, \varepsilon}^{2} e^{u_{k+1, \varepsilon}^{2}+\left|u_{k+1, \varepsilon}\right|^{1+\varepsilon}} d x \\
& \leq \lambda e^{\left\|u_{k+1, \varepsilon}\right\|_{\infty}^{2}+\left\|u_{k+1, \varepsilon}\right\|_{\infty}^{1+\varepsilon}} \int_{B} u_{k+1, \varepsilon}^{2} d x \rightarrow 0
\end{aligned}
$$

as $\varepsilon \rightarrow 0$, a contradiction. As a consequence, setting $\left\|u_{k+1, \varepsilon}\right\|_{\infty}=u_{k, \varepsilon}\left(r_{k, \varepsilon}^{*}\right)$ with a value $r_{k, \varepsilon}^{*} \in\left(r_{k, \varepsilon}, 1\right)$, we get from Lemma 2.3 that

$$
\left\|u_{k, \varepsilon}\right\| \geq c_{2}^{-1}\left|u_{k, \varepsilon}\left(r_{k, \varepsilon}^{*}\right)\right|\left(r_{k, \varepsilon}^{*}\right)^{\frac{1}{2}} \geq c_{2}^{-1}\left|u_{k, \varepsilon}\left(r_{k, \varepsilon}^{*}\right)\right| r_{k, \varepsilon}^{\frac{1}{2}} \rightarrow \infty
$$

as $\varepsilon \rightarrow 0$ since $r_{k}>0$, which contradicts Lemma 2.2. This shows the claim. Especially we get $0 \leq r_{1} \leq \cdots \leq r_{k} \in(0,1)$. Now recalling that 
$u$ is a radial solution and $\lambda<\lambda_{\mathrm{AY}}$ and then, noting $(-1)^{k} u_{k+1} \geq 0$ is nontrivial and $(-1)^{k-1} u_{k} \geq 0$, we must have $u_{k}=0$. Then, the maximum principle yields $r_{k}=r_{k+1}$. Finally, repeating the argument above, we get $\sup _{r \in\left(r_{k-1, \varepsilon}, r_{k, \varepsilon}\right)} u_{k, \varepsilon}(r) \rightarrow \infty$ as $\varepsilon \rightarrow 0$ and then Lemmas 2.2 and 2.3 lead us to the contradiction. This finishes the proof.

Finally, let us investigate the limit value of the energy $I_{\varepsilon}\left(u_{\varepsilon}\right)$ more precisely.

Lemma 2.5. We get

$$
\lim _{\varepsilon \rightarrow 0} I_{\varepsilon}\left(u_{i, \varepsilon}\right)=2 \pi
$$

for all $i=1,2, \cdots, k$. Furthermore, we obtain

$$
\limsup _{\varepsilon \rightarrow 0} I_{\varepsilon}\left(u_{k+1, \varepsilon}\right)=I_{0}\left(u_{0}\right) .
$$

Proof. Choose $i=1,2, \cdots, k$. We first claim

$$
\liminf _{\varepsilon \rightarrow 0} I_{\varepsilon}\left(u_{i, \varepsilon}\right) \geq 2 \pi \text {. }
$$

Indeed, let $\tilde{u}_{0, \varepsilon} \in H_{0}^{1}(B)$ be a positive solution of (1.9) with $B$ replaced by $B_{r_{i, \varepsilon}}$ which satisfies

$$
I_{\varepsilon}\left(\tilde{u}_{0, \varepsilon}\right)=\inf \left\{\left.I_{\varepsilon}(u)\left|u \in H_{0}^{1}\left(B_{r_{i, \varepsilon}}\right), \int_{B_{r_{i, \varepsilon}}}\right| \nabla u\right|^{2} d x=\int_{B_{r_{i, \varepsilon}}} f_{\varepsilon}(u) u d x .\right\} .
$$

The existence of $\tilde{u}_{0, \varepsilon}$ is ensured by 2]. Then we have $I_{\varepsilon}\left(u_{i, \varepsilon}\right) \geq I_{\varepsilon}\left(\tilde{u}_{0, \varepsilon}\right)$. Hence it suffices to show $\liminf _{\varepsilon \rightarrow 0} I_{\varepsilon}\left(\tilde{u}_{0, \varepsilon}\right) \geq 2 \pi$. Now we assume, on the contrary, $\liminf \inf _{\varepsilon \rightarrow 0} I_{\varepsilon}\left(\tilde{u}_{0, \varepsilon}\right)<2 \pi$. Set $v_{i, \varepsilon}(x)=\tilde{u}_{0, \varepsilon}\left(r_{i, \varepsilon} x\right)$. Then $v=v_{i, \varepsilon}$ satisfies

$$
\left\{\begin{array}{l}
-\Delta v=\lambda r_{i, \varepsilon}^{2} v e^{v^{2}+v^{1+\varepsilon}}, v>0 \text { in } B, \\
v=0 \text { on } \partial B .
\end{array}\right.
$$

We define the energy associated to (2.4).

$$
J_{\varepsilon}(v)=\int_{B}|\nabla v|^{2} d x-r_{i, \varepsilon}^{2} \int_{B} F_{\varepsilon}(v) d x\left(v \in H_{0}^{1}(B)\right) .
$$

Then we have $I_{\varepsilon}\left(\tilde{u}_{0, \varepsilon}\right)=J_{\varepsilon}\left(v_{i, \varepsilon}\right)$ and thus, $\liminf _{\varepsilon \rightarrow 0} J_{\varepsilon}\left(v_{i, \varepsilon}\right)<2 \pi$. In particular, we have a sequence $\left(\varepsilon_{n}\right)$ such that $\varepsilon_{n} \rightarrow 0$ as $n \rightarrow \infty$ and $c:=$ $\lim _{n \rightarrow \infty} J_{\varepsilon_{n}}\left(v_{i, \varepsilon_{n}}\right)<2 \pi$. Notice that Lemma 2.2 ensures $c>0$. Then, noting $J_{\varepsilon_{n}}^{\prime}\left(v_{i, \varepsilon_{n}}\right)=0$ and Lemma $\mathbf{A . 3}$ in Appendix $\mathrm{A}$, we can find a function $v_{0} \in H_{0}^{1}(\Omega)$ such that $v_{i, \varepsilon_{n}} \rightarrow v_{0}$ in $H_{0}^{1}(\Omega)$ as $n \rightarrow \infty$ up to subsequences. Lastly, using (2.4), we get

$$
\left\{\begin{array}{l}
-\Delta v_{0}=0, v_{0} \geq 0 \text { in } B \\
v_{0}=0 \text { on } \partial B
\end{array}\right.
$$

Then, the maximum principle shows $v_{0}=0$. But this contradicts $c>0$. Next let us show

$$
\limsup _{\varepsilon \rightarrow 0} I_{\varepsilon}\left(u_{i, \varepsilon}\right) \leq 2 \pi, \text { and } \limsup _{\varepsilon \rightarrow 0} I_{\varepsilon}\left(u_{k+1, \varepsilon}\right)=I_{0}\left(u_{0}\right) .
$$


In fact, we get by Lemma 2.1 and (2.3) that

$$
2 \pi k+I_{0}\left(u_{0}\right) \geq \limsup _{\varepsilon \rightarrow 0} I_{\varepsilon}\left(u_{\varepsilon}\right) \geq 2 \pi k+\limsup _{\varepsilon \rightarrow 0} I_{\varepsilon}\left(u_{k+1, \varepsilon}\right)
$$

which implies $I_{0}\left(u_{0}\right) \geq \lim \sup _{\varepsilon \rightarrow 0} I_{\varepsilon}\left(u_{k+1, \varepsilon}\right)$. Furthermore, let $u_{0, \varepsilon}$ be the least energy solution of (1.9) obtained by [2. It follows that $I_{0}\left(u_{0}\right) \geq$ $\lim \sup _{\varepsilon \rightarrow 0} I_{\varepsilon}\left(u_{k+1, \varepsilon}\right) \geq \lim \sup _{\varepsilon \rightarrow 0} I_{\varepsilon}\left(u_{0, \varepsilon}\right)$. We claim $\lim \sup _{\varepsilon \rightarrow 0} I_{\varepsilon}\left(u_{0, \varepsilon}\right) \geq$ $I_{0}\left(u_{0}\right)$. If not, we have a sequence $\left(\varepsilon_{n}\right)$ such that $\varepsilon_{n} \rightarrow 0$ as $n \rightarrow \infty$ and $\lim _{n \rightarrow \infty} I_{\varepsilon_{n}}\left(u_{0, \varepsilon}\right)<I_{0}\left(u_{0}\right)$. Note $I_{0}\left(u_{0}\right) \in(0,2 \pi)$. Then from Lemma A.3. we deduce, by subtracting a subsequence if necessary, $u_{0, \varepsilon_{n}} \rightarrow \tilde{u}_{0}$ in $H_{0}^{1}(B)$ as $n \rightarrow \infty$ and further, $\tilde{u}_{0}$ is a nontrivial solution of (1.7) with $I_{0}\left(\tilde{u}_{0}\right) \in\left(0, I_{0}\left(u_{0}\right)\right)$. But as $\tilde{u}_{0} \in \mathcal{N}_{0}$, we obtain a contradiction by the definition of $u_{0}$. This proves the claim. Now again arguing as the beginning, we get

$$
2 \pi k+I_{0}\left(u_{0}\right) \geq \limsup _{\varepsilon \rightarrow 0} I_{\varepsilon}\left(u_{\varepsilon}\right) \geq 2 \pi(k-1)+\limsup _{\varepsilon \rightarrow 0} I_{\varepsilon}\left(u_{i, \varepsilon}\right)+I_{0}\left(u_{0}\right) .
$$

This completes (2.5). As a consequence, (2.3) and (2.5) finish the proof.

Lemma 2.6. We have

$$
\lim _{\varepsilon \rightarrow 0} I_{\varepsilon}\left(u_{k+1, \varepsilon}\right)=I_{0}\left(u_{0}\right) .
$$

Proof. Since $\liminf \operatorname{in}_{\varepsilon \rightarrow 0} I_{\varepsilon}\left(u_{k+1, \varepsilon}\right) \leq I_{0}\left(u_{0}\right)$, arguing as in the previous proof, we can get

$$
\liminf _{\varepsilon \rightarrow 0} I_{\varepsilon}\left(u_{k+1, \varepsilon}\right)=I_{0}\left(u_{0}\right) .
$$

Then combining this together with the final assertion in the previous lemma, we complete the proof.

\section{Behavior of $u_{\varepsilon}$ IN THE BALL $B_{r_{1, \varepsilon}}$}

Let us start our main argument with studying the behavior on a ball. To this end, we first observe that $u_{1, \varepsilon}=\left.u_{\varepsilon}\right|_{B_{r_{1, \varepsilon}}}$ is a solution to

$$
\left\{\begin{array}{l}
-\Delta u=\lambda u e^{u^{2}+|u|^{1+\varepsilon}}, u>0 \text { in } B_{r_{1, \varepsilon}} \\
u=0 \text { on } \partial B_{r_{1, \varepsilon}},
\end{array}\right.
$$

for $\varepsilon>0$. Then the results in 14 shows that $u_{1, \varepsilon}$ is radial and $\left\|u_{1, \varepsilon}\right\|_{L^{\infty}\left(B_{r_{1, \varepsilon}}\right)}=$ $u_{1, \varepsilon}(0)$. Next we see that $v_{1, \varepsilon}(x):=u_{\varepsilon}\left(r_{1, \varepsilon} x\right)\left(x \in B_{1}\right)$ is a solution of

$$
\left\{\begin{array}{l}
-\Delta v=\lambda r_{1, \varepsilon}^{2} v e^{v^{2}+|v|^{1+\varepsilon}}, v>0 \text { in } B \\
v=0 \text { on } \partial B,
\end{array}\right.
$$

for $\varepsilon>0$ and $\left\|v_{1, \varepsilon}\right\|_{L^{\infty}(B)}=v_{\varepsilon}(0)$. Notice $\lambda r_{1, \varepsilon}^{2} \rightarrow 0$ as $\varepsilon \rightarrow 0$ by Lemma 2.4. Furthermore, by Lemma 2.5, we get

$$
J_{\varepsilon}\left(v_{\varepsilon}\right):=\frac{1}{2} \int_{B}\left|\nabla v_{\varepsilon}\right|^{2} d x-r_{1, \varepsilon}^{2} \int_{B} F_{\varepsilon}\left(v_{\varepsilon}\right) d x \rightarrow 2 \pi,
$$

as $\varepsilon \rightarrow 0$. We have the following 
Proposition 3.1. We get $v_{1, \varepsilon} \rightarrow 0$ weakly in $H_{0}^{1}(B), v_{1, \varepsilon}(0) \rightarrow \infty$ and

$$
\int_{B}\left|\nabla v_{1, \varepsilon}\right|^{2} d x \rightarrow 4 \pi
$$

as $\varepsilon \rightarrow 0$. Furthermore, let $\gamma_{1, \varepsilon}>0$ be such that $2 \lambda r_{1, \varepsilon}^{2} v_{1, \varepsilon}(0)^{2} e^{v_{1, \varepsilon}(0)^{2}+v_{1, \varepsilon}(0)^{1+\varepsilon}} \gamma_{1, \varepsilon}^{2}=$ 1. Then we have $\gamma_{1, \varepsilon} \rightarrow 0$ and

$$
2 v_{1, \varepsilon}(0)\left(v_{1, \varepsilon}\left(\gamma_{1, \varepsilon} x\right)-v_{1, \varepsilon}(0)\right) \rightarrow \log \frac{1}{\left(1+|x|^{2} / 8\right)^{2}} \text { in } C_{l o c}^{2}\left(\mathbb{R}^{2}\right),
$$

as $\varepsilon \rightarrow 0$.

Proof. It is a direct consequence of Theorem 2 in 3 .

Corollary 3.2. We obtain $u_{1, \varepsilon} \rightarrow 0$ weakly in $H_{0}^{1}(B), u_{1, \varepsilon}(0) \rightarrow \infty$ and

$$
\int_{B_{r_{1, \varepsilon}}}\left|\nabla u_{1, \varepsilon}\right|^{2} d x \rightarrow 4 \pi
$$

and

$$
I_{\varepsilon}\left(u_{1, \varepsilon}\right) \rightarrow 2 \pi,
$$

as $\varepsilon \rightarrow 0$. Furthermore, let $\delta_{1, \varepsilon}=r_{1, \varepsilon} \gamma_{1, \varepsilon}>0$. Then we have $\delta_{1, \varepsilon} \rightarrow 0$ and

$$
2 u_{\varepsilon}(0)\left(u_{\varepsilon}\left(\delta_{1, \varepsilon} x\right)-u_{\varepsilon}(0)\right) \rightarrow \log \frac{1}{\left(1+|x|^{2} / 8\right)^{2}} \text { in } C_{l o c}^{2}\left(\mathbb{R}^{2}\right),
$$

as $\varepsilon \rightarrow 0$.

Proof. The proof follows from Proposition 3.1 and Lemma 2.5.

\section{Behavior of $u_{\varepsilon}$ ON ANNUli}

We next investigate the behavior of $u_{\varepsilon}$ on annuli. Fix $i \in\{2, \cdots, k\}$ and set $u_{i, \varepsilon}:=\left.u_{\varepsilon}\right|_{B\left(r_{i-1, \varepsilon}, r_{i, \varepsilon}\right)}$. Then $u_{i, \varepsilon} \in H_{0}^{1}(B)$ by zero extension. Since $u_{i, \varepsilon}$ is radial, we may assume it satisfies

$$
\begin{cases}-u_{i, \varepsilon}^{\prime \prime}-\frac{1}{r} u_{i, \varepsilon}^{\prime}=\lambda u_{i, \varepsilon} e^{u_{i, \varepsilon}^{2}+u_{i, \varepsilon}^{1+\varepsilon},} & \text { in }\left(r_{i-1, \varepsilon}, r_{i, \varepsilon}\right) \\ u_{i, \varepsilon}>0 & \text { in }\left(r_{i-1, \varepsilon}, r_{i, \varepsilon}\right) \\ u_{i, \varepsilon}\left(r_{i-1, \varepsilon}\right)=u_{i, \varepsilon}\left(r_{i, \varepsilon}\right)=0 . & \end{cases}
$$

Now we have the following result.

Proposition 4.1. We get $u_{i, \varepsilon} \rightarrow 0$ weakly in $H_{0}^{1}(B)$,

$$
\int_{B\left(r_{i-1, \varepsilon}, r_{i, \varepsilon}\right)}\left|\nabla u_{i, \varepsilon}\right|^{2} d x \rightarrow 4 \pi
$$

and

$$
I_{\varepsilon}\left(u_{i, \varepsilon}\right) \rightarrow 2 \pi,
$$

as $\varepsilon \rightarrow 0$. Moreover, let us denote by $M_{i, \varepsilon} r_{i, \varepsilon} \in\left(r_{i-1, \varepsilon}, r_{i, \varepsilon}\right)$ with $M_{i, \varepsilon}<1$, the point such that $\left\|u_{i, \varepsilon}\right\|_{L^{\infty}\left(r_{i-1, \varepsilon}, r_{i, \varepsilon}\right)}=u_{i, \varepsilon}\left(M_{i, \varepsilon} r_{i, \varepsilon}\right)$. Then if we set $\delta_{i, \varepsilon}=$ $\gamma_{i, \varepsilon} r_{i, \varepsilon}>0$ with

$$
2 \lambda\left\|u_{i, \varepsilon}\right\|_{L^{\infty}\left(r_{i-1, \varepsilon}, r_{i, \varepsilon}\right)}^{2} e^{\left\|u_{i, \varepsilon}\right\|_{L^{\infty}\left(r_{i-1, \varepsilon}, r_{i, \varepsilon}\right)}^{2}+\left\|u_{i, \varepsilon}\right\|_{L^{\infty}\left(r_{i-1, \varepsilon}, r_{i, \varepsilon}\right)}^{1+\varepsilon}} r_{i, \varepsilon}^{2} \gamma_{i, \varepsilon}^{2}=1,
$$


we get $\delta_{i, \varepsilon} \rightarrow 0$ and further,

$$
\begin{aligned}
2\left\|u_{i, \varepsilon}\right\|_{L^{\infty}\left(r_{i-1, \varepsilon}, r_{i, \varepsilon}\right)}\left(u_{i, \varepsilon}\left(M_{\varepsilon} r_{i, \varepsilon}+\delta_{i, \varepsilon} r\right)\right. & \left.-\left\|u_{i, \varepsilon}\right\|_{L^{\infty}\left(r_{i-1, \varepsilon}, r_{i, \varepsilon}\right)}\right) \\
& \rightarrow \log \frac{1}{\left(1+r^{2} / 8\right)^{2}} \text { in } C_{l o c}^{2}\left(\mathbb{R}^{+}\right),
\end{aligned}
$$

as $\varepsilon \rightarrow 0$.

In the following, we set $M_{\varepsilon}:=M_{i, \varepsilon}$ for simplicity. We get the following.

Lemma 4.2. $u_{i, \varepsilon}\left(M_{\varepsilon} r_{i, \varepsilon}\right) \rightarrow+\infty$ as $\varepsilon \rightarrow 0$.

Proof. Integrating (4.1) we get

$$
\begin{aligned}
& \int_{r_{i-1, \varepsilon}}^{r_{i, \varepsilon}}\left(u_{i, \varepsilon}^{\prime}\right)^{2} r d r=\lambda \int_{r_{i-1, \varepsilon}}^{r_{i, \varepsilon}} u_{i, \varepsilon}^{2} e^{u_{i, \varepsilon}^{2}+u_{i, \varepsilon}^{1+\varepsilon}} r d r \\
& \leq \lambda e^{u_{i, \varepsilon}^{2}\left(M_{\varepsilon} r_{i, \varepsilon}\right)+u_{i, \varepsilon}^{1+\varepsilon}\left(M_{\varepsilon} r_{i, \varepsilon}\right)} \int_{r_{i-1, \varepsilon}}^{r_{i, \varepsilon}} u_{i, \varepsilon}^{2} r d r
\end{aligned}
$$

(using the Poincare inequality) $\leq \lambda \frac{e^{u_{i, \varepsilon}^{2}\left(M_{\varepsilon} r_{i, \varepsilon}\right)+u_{i, \varepsilon}^{1+\varepsilon}\left(M_{\varepsilon} r_{i, \varepsilon}\right)}}{\lambda_{1}\left(r_{i-1, \varepsilon}, r_{i, \varepsilon}\right)} \int_{r_{i-1, \varepsilon}}^{r_{i, \varepsilon}}\left(u_{i, \varepsilon}^{\prime}\right)^{2} r d r$,

where $\lambda_{1}\left(r_{i-1, \varepsilon}, r_{i, \varepsilon}\right)$ is the first eigenvalue of the operator $-u^{\prime \prime}-\frac{1}{r} u^{\prime}$ in $\left(r_{i-1, \varepsilon}, r_{i, \varepsilon}\right)$. Since $r_{i-1, \varepsilon}, r_{i, \varepsilon} \rightarrow 0$ we get that $\lambda_{1}\left(r_{i-1, \varepsilon}, r_{i, \varepsilon}\right) \rightarrow+\infty$ as $\varepsilon \rightarrow 0$. This gives the claim.

Now, let us consider the scaled function, $v_{\varepsilon}:\left(\frac{r_{i-1, \varepsilon}}{r_{i, \varepsilon}}, 1\right) \rightarrow \mathbb{R}$ defined as

$$
v_{\varepsilon}(r)=u_{i, \varepsilon}\left(r_{i, \varepsilon} r\right)
$$

which satisfies

$$
\begin{cases}-v_{\varepsilon}^{\prime \prime}-\frac{1}{r} v_{\varepsilon}^{\prime}=\lambda r_{i, \varepsilon}^{2} v_{\varepsilon} e^{v_{\varepsilon}^{2}+v_{\varepsilon}^{1+\varepsilon}} & \text { in }\left(\frac{r_{i-1, \varepsilon}}{r_{i, \varepsilon}}, 1\right) \\ v_{\varepsilon}>0 & \text { in }\left(\frac{r_{i-1, \varepsilon}}{r_{i, \varepsilon}}, 1\right) \\ v_{\varepsilon}\left(\frac{r_{i-1, \varepsilon}}{r_{i, \varepsilon}}\right)=v_{\varepsilon}(1)=0 . & \end{cases}
$$

Set

$$
r_{\varepsilon}=\frac{r_{i-1, \varepsilon}}{r_{i, \varepsilon}}
$$

Then we have the following local behavior.

Lemma 4.3. Choose $M_{\varepsilon} \in\left(r_{\varepsilon}, 1\right)$ as in Proposition 4.1. Then if we set $\gamma_{i, \varepsilon}>0$ so that

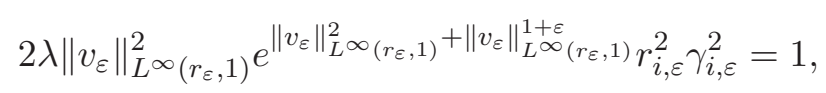

we get $\gamma_{i, \varepsilon} \rightarrow 0$ and

$2\left\|v_{\varepsilon}\right\|_{L^{\infty}\left(r_{\varepsilon}, 1\right)}\left(v_{\varepsilon}\left(M_{\varepsilon}+\gamma_{i, \varepsilon} r\right)-\left\|v_{\varepsilon}\right\|_{L^{\infty}\left(r_{\varepsilon}, 1\right)}\right) \rightarrow z(r)=\log \frac{1}{\left(1+r^{2} / 8\right)^{2}}$ in $C_{l o c}^{2}(0,+\infty)$, as $\varepsilon \rightarrow 0$. 
Proof. Let $v_{\varepsilon}, r_{\varepsilon}$ and $M_{\varepsilon} \in\left(r_{\varepsilon}, 1\right)$ as above. For $\gamma_{\varepsilon}>0$, which will be chosen later, we define the scaled function

$$
z_{\varepsilon}(r)=2 v_{\varepsilon}\left(M_{\varepsilon}\right)\left(v_{\varepsilon}\left(M_{\varepsilon}+\gamma_{\varepsilon} r\right)-v_{\varepsilon}\left(M_{\varepsilon}\right)\right) .
$$

We have that $z_{\varepsilon}$ solves the equation,

$$
\left\{\begin{array}{l}
-z_{\varepsilon}^{\prime \prime}-\frac{1}{\frac{M_{\varepsilon}}{\gamma_{\varepsilon}}+r} z_{\varepsilon}^{\prime}=2 \lambda \gamma_{\varepsilon}^{2} r_{i, \varepsilon}^{2} e^{v_{\varepsilon}^{2}\left(M_{\varepsilon}\right)+v_{\varepsilon}^{1+\varepsilon}\left(M_{\varepsilon}\right)} v_{\varepsilon}^{2}\left(M_{\varepsilon}\right)\left(\frac{z_{\varepsilon}}{2 v_{\varepsilon}^{2}\left(M_{\varepsilon}\right)}+1\right) \\
\quad \times e^{\left\{z_{\varepsilon}\left(\frac{z_{\varepsilon}}{4 v_{\varepsilon}^{2}\left(M_{\varepsilon}\right)}+1\right)+v_{\varepsilon}^{1+\varepsilon}\left(M_{\varepsilon}\right)\left(\left|\frac{z_{\varepsilon}}{2 v_{\varepsilon}^{2}\left(M_{\varepsilon}\right)}+1\right|^{1+\varepsilon}-1\right)\right\}} \text { in }\left(\frac{r_{\varepsilon}-M_{\varepsilon}}{\gamma_{\varepsilon}}, \frac{1-M_{\varepsilon}}{\gamma_{\varepsilon}}\right), \\
z_{\varepsilon}(r) \leq 0, z_{\varepsilon}(0)=z_{\varepsilon}^{\prime}(0)=0, \\
z_{\varepsilon}\left(\frac{r_{\varepsilon}-M_{\varepsilon}}{\gamma_{\varepsilon}}\right)=z_{\varepsilon}\left(\frac{1-M_{\varepsilon}}{\gamma_{\varepsilon}}\right)=-2 v_{\varepsilon}^{2}\left(M_{\varepsilon}\right) \rightarrow-\infty(\varepsilon \rightarrow 0) .
\end{array}\right.
$$

So setting

$$
2 \lambda \gamma_{\varepsilon}^{2} r_{i, \varepsilon}^{2} e^{v_{\varepsilon}^{2}\left(M_{\varepsilon}\right)+v_{\varepsilon}^{1+\varepsilon}\left(M_{\varepsilon}\right)} v_{\varepsilon}^{2}\left(M_{\varepsilon}\right)=1
$$

we get

$$
\left\{\begin{array}{l}
-z_{\varepsilon}^{\prime \prime}-\frac{1}{\frac{M_{\varepsilon}}{\gamma_{\varepsilon}}+r} z_{\varepsilon}^{\prime}=\left(\frac{z_{\varepsilon}}{2 v_{\varepsilon}^{2}\left(M_{\varepsilon}\right)}+1\right) e^{\left\{z_{\varepsilon}\left(\frac{z_{\varepsilon}}{4 v_{\varepsilon}^{2}\left(M_{\varepsilon}\right)}+1\right)+v_{\varepsilon}^{1+\varepsilon}\left(M_{\varepsilon}\right)\left(\left|\frac{z_{\varepsilon}}{2 v_{\varepsilon}^{2}\left(M_{\varepsilon}\right)}+1\right|^{1+\varepsilon}-1\right)\right\}} \\
z_{\varepsilon}(r) \leq 0, z_{\varepsilon}(0)=z_{\varepsilon}^{\prime}(0)=0, \\
z_{\varepsilon}\left(\frac{r_{\varepsilon}-M_{\varepsilon}}{\gamma_{\varepsilon}}\right)=z_{\varepsilon}\left(\frac{1-M_{\varepsilon}}{\gamma_{\varepsilon}}, \frac{1-M_{\varepsilon}}{\gamma_{\varepsilon}}\right),=-2 v_{\varepsilon}^{2}\left(M_{\varepsilon}\right) \rightarrow-\infty(\varepsilon \rightarrow 0) .
\end{array}\right.
$$

Note that $\gamma_{\varepsilon} \rightarrow 0$ as $\varepsilon \rightarrow 0$. Actually, multiplying (4.2) by $v_{\varepsilon} r$ and integrating over $(0,1)$, we get

$$
\begin{aligned}
& \int_{0}^{1}\left(v_{\varepsilon}^{\prime}\right)^{2} r d r=\lambda r_{i, \varepsilon}^{2} \int_{0}^{1} v_{\varepsilon}^{2} e^{v_{\varepsilon}^{2}+v_{\varepsilon}^{1+\varepsilon}} r d r \\
& \leq \lambda r_{i, \varepsilon}^{2} e^{v_{\varepsilon}^{2}\left(M_{\varepsilon}\right)+v_{\varepsilon}^{1+\varepsilon}\left(M_{\varepsilon}\right)} \int_{0}^{1} v_{\varepsilon}^{2} r d r
\end{aligned}
$$

(applying the Poincare inequality) $\leq \frac{\lambda}{\lambda_{1}} r_{i, \varepsilon}^{2} e^{v_{\varepsilon}^{2}\left(M_{\varepsilon}\right)+v_{\varepsilon}^{1+\varepsilon}\left(M_{\varepsilon}\right)} \int_{0}^{1}\left(v_{\varepsilon}^{\prime}\right)^{2} r d r$.

This shows

$$
r_{i, \varepsilon}^{2} e^{v_{\varepsilon}^{2}\left(M_{\varepsilon}\right)+v_{\varepsilon}^{1+\varepsilon}\left(M_{\varepsilon}\right)} \geq C>0
$$

for some constant $C>0$ and small $\varepsilon>0$. Then noting our choice of $\gamma_{\varepsilon}$ and Lemma 4.2, we prove the claim. Moreover we clearly have that $\lim _{\varepsilon \rightarrow 0} \frac{1-M_{\varepsilon}}{\gamma_{\varepsilon}} \rightarrow \infty, \lim _{\varepsilon \rightarrow 0} \frac{M_{\varepsilon}-r_{\varepsilon}}{\gamma_{\varepsilon}}=l \in[0, \infty]$ and $\lim _{\varepsilon \rightarrow 0} \frac{M_{\varepsilon}}{\gamma_{\varepsilon}}=m \in[l, \infty]$. Now let us show that for any compact subset $K \subset \subset(-l, \infty)([0, \infty)$ if $l=0)$, there exists a constant $C>0$ which is independent of $\varepsilon$ such that

$$
\left\|z_{\varepsilon}\right\|_{C^{1}(K)} \leq C \text {. }
$$

Indeed, from (4.4), we get that $-z_{\varepsilon}^{\prime \prime}-\frac{1}{\frac{M_{\varepsilon}}{\gamma_{\varepsilon}}+r} z_{\varepsilon}^{\prime} \leq 1$. First assume $l>0$ and choose any $K \subset \subset(-l, 0]$. We may suppose $K \subset \subset\left(\frac{r_{\varepsilon}-M_{\varepsilon}}{\gamma_{\varepsilon}}, 0\right]$ for small $\varepsilon>0$. Define $a=\min K<0$ and set $C_{\varepsilon}=\frac{M_{\varepsilon}}{\gamma_{\varepsilon}}$. Then, for any $r \in K$, we derive,

$$
-\left[z_{\varepsilon}^{\prime}(r)\left(C_{\varepsilon}+r\right)\right]^{\prime} \leq C_{\varepsilon}+r .
$$


Integrating between $r$ and 0 we obtain

$$
z_{\varepsilon}^{\prime}(r)\left(C_{\varepsilon}+r\right) \leq-\left(C_{\varepsilon} r+\frac{1}{2} r^{2}\right)
$$

Since $C_{\varepsilon}+r>0$ for small $\varepsilon>0$, we show

$$
z_{\varepsilon}^{\prime}(r) \leq-\frac{C_{\varepsilon} r+\frac{1}{2} r^{2}}{C_{\varepsilon}+r} \text { and thus, } z_{\varepsilon}(r) \geq \int_{r}^{0} \frac{C_{\varepsilon} s+\frac{1}{2} s^{2}}{C_{\varepsilon}+s} d s
$$

for small $\varepsilon>0$. If we set $G_{\varepsilon}(s)=\frac{C_{\varepsilon} s+\frac{1}{2} s^{2}}{C_{\varepsilon}+s}$, we get that $G_{\varepsilon}^{\prime}(s) \geq 0$ for all $s \in K$. So we find that $G_{\varepsilon}(s) \geq G_{\varepsilon}(a)$ for all $s \in K$. Now, if $C_{\varepsilon} \rightarrow \infty$ as $\varepsilon \rightarrow 0$, we get $G_{\varepsilon}(a) \geq-2|a|$ for small $\varepsilon>0$. If $C_{\varepsilon}$ is bounded, we get a constant $c_{0}>0$ such that $G_{\varepsilon}(a) \geq-c_{0}$ for small $\varepsilon>0$. This implies that there exists a constant $c_{1}>0$ such that

$$
z_{\varepsilon}^{\prime}(r) \leq c_{1} \text { and thus, } z_{\varepsilon} \geq c_{1} a \text { on } K,
$$

for all small $\varepsilon$. Hence we have a constant $C>0$ such that $\left\|z_{\varepsilon}\right\|_{C^{1}(K)} \leq C$ uniformly for small $\varepsilon>0$. On the other hand, for any compact subset $K \subset \subset[0, \infty)$, repeating the same argument as above, we get the desired uniform bound for $\left\|z_{\varepsilon}\right\|_{C^{1}(K)}$. This proves the claim. Consequently, we may pass to the limit in the equation (4.4). Now let us discuss the "limit domain". We have three possibilities,
1. $\frac{r_{\varepsilon}-M_{\varepsilon}}{\gamma_{\varepsilon}} \rightarrow-\infty$,
2. $\frac{r_{\varepsilon}-M_{\varepsilon}}{\gamma_{\varepsilon}} \rightarrow-l<0$.
3. $\frac{r_{\varepsilon}-M_{\varepsilon}}{\gamma_{\varepsilon}} \rightarrow 0$,

We will show that only case 3 occurs.

Case 1: $\frac{r_{\varepsilon}-M_{\varepsilon}}{\gamma_{\varepsilon}} \rightarrow-\infty$ cannot occur

First we note that in this case we have that $\frac{M_{\varepsilon}}{\gamma_{\varepsilon}} \rightarrow+\infty$. Then, passing to the limit in (4.4), we get that there exists a function $z$ which satisfies $z_{\varepsilon} \rightarrow z$ in $C_{\text {loc }}^{2}(\mathbb{R})$ and

$$
\left\{\begin{array}{l}
-z^{\prime \prime}=e^{z} \quad \text { in } \mathbb{R} \\
z(0)=z^{\prime}(0)=0 .
\end{array}\right.
$$


Hence $z(s)=\log \frac{4 e^{\sqrt{2} s}}{\left(1+e^{\sqrt{2} s}\right)^{2}}$. So we have that

$$
\begin{aligned}
& \int_{r_{\varepsilon}}^{1}\left|v_{\varepsilon}^{\prime}\right|^{2} r d r=\lambda r_{i, \varepsilon}^{2} \int_{r_{\varepsilon}}^{1} v_{\varepsilon}^{2} e^{v_{\varepsilon}^{2}+v_{\varepsilon}^{1+\varepsilon}} r d r \\
& =\lambda r_{i, \varepsilon}^{2} \gamma_{\varepsilon} e^{v_{\varepsilon}^{2}\left(M_{\varepsilon}\right)+v_{\varepsilon}^{1+\varepsilon}\left(M_{\varepsilon}\right)} v_{\varepsilon}^{2}\left(M_{\varepsilon}\right) \\
& \times \int_{\frac{r_{\varepsilon}-M_{\varepsilon}}{\gamma_{\varepsilon}}}^{\frac{1-M_{\varepsilon}}{\gamma_{\varepsilon}}}\left(\frac{z_{\varepsilon}(r)}{2 v_{\varepsilon}^{2}\left(M_{\varepsilon}\right)}+1\right)^{2} e^{z_{\varepsilon}(r)\left(\frac{z_{\varepsilon}(r)}{4 v_{\varepsilon}^{2}\left(M_{\varepsilon}\right)}+1\right)+v_{\varepsilon}^{1+\varepsilon}\left(M_{\varepsilon}\right)\left(\left|\frac{z_{\varepsilon}(r)}{2 v_{\varepsilon}^{2}\left(M_{\varepsilon}\right)}+1\right|^{1+\varepsilon}-1\right)}\left(M_{\varepsilon}+\gamma_{\varepsilon} r\right) d r \\
& \geq \lambda r_{i, \varepsilon}^{2} \gamma_{\varepsilon} M_{\varepsilon} e^{v_{\varepsilon}^{2}\left(M_{\varepsilon}\right)+v_{\varepsilon}^{1+\varepsilon}\left(M_{\varepsilon}\right)} v_{\varepsilon}^{2}\left(M_{\varepsilon}\right) \\
& \times \int_{0}^{\frac{1-M_{\varepsilon}}{\gamma_{\varepsilon}}}\left(\frac{z_{\varepsilon}(r)}{2 v_{\varepsilon}^{2}\left(M_{\varepsilon}\right)}+1\right)^{2} e^{z_{\varepsilon}(r)\left(\frac{z_{\varepsilon}(r)}{4 v_{\varepsilon}^{2}\left(M_{\varepsilon}\right)}+1\right)+v_{\varepsilon}^{1+\varepsilon}\left(M_{\varepsilon}\right)\left(\left|\frac{z_{\varepsilon}(r)}{2 v_{\varepsilon}^{2}\left(M_{\varepsilon}\right)}+1\right|^{1+\varepsilon}-1\right)} d r \\
& =\frac{M_{\varepsilon}}{2 \gamma_{\varepsilon}} \int_{0}^{\frac{1-M_{\varepsilon}}{\gamma_{\varepsilon}}}\left(\frac{z_{\varepsilon}(r)}{2 v_{\varepsilon}^{2}\left(M_{\varepsilon}\right)}+1\right)^{2} e^{z_{\varepsilon}(r)\left(\frac{z_{\varepsilon}(r)}{4 v_{\varepsilon}^{2}\left(M_{\varepsilon}\right)}+1\right)+v_{\varepsilon}^{1+\varepsilon}\left(M_{\varepsilon}\right)\left(\left|\frac{z_{\varepsilon}(r)}{2 v_{\varepsilon}^{2}\left(M_{\varepsilon}\right)}+1\right|^{1+\varepsilon}-1\right)} d r .
\end{aligned}
$$

Here Fatou's lemma implies that

$$
\begin{array}{r}
\liminf _{\varepsilon \rightarrow 0} \int_{0}^{\frac{1-M_{\varepsilon}}{\gamma_{\varepsilon}}}\left(\frac{z_{\varepsilon}(r)}{2 v_{\varepsilon}\left(M_{\varepsilon}\right)}+1\right)^{2} e^{z_{\varepsilon}(r)\left(\frac{z_{\varepsilon}(r)}{4 v_{\varepsilon}^{2}\left(M_{\varepsilon}\right)}+1\right)+v_{\varepsilon}^{1+\varepsilon}\left(M_{\varepsilon}\right)}\left(\left|\frac{z_{\varepsilon}(r)}{2 v_{\varepsilon}^{2}\left(M_{\varepsilon}\right)}+1\right|^{1+\varepsilon}-1\right) \\
\\
\geq \int_{0}^{+\infty} e^{z(s)} d r>0 .
\end{array}
$$

Therefore by Lemma 2.2, we deduce a contradiction since $M_{\varepsilon} / \gamma_{\varepsilon} \rightarrow \infty$ as $\varepsilon \rightarrow 0$. This ends Case 1 .

\section{Case 2: $\frac{r_{\varepsilon}-M_{\varepsilon}}{\gamma_{\varepsilon}} \rightarrow-l<0$ cannot occur}

Noting $m:=\lim _{\varepsilon \rightarrow 0} \frac{M_{\varepsilon}}{\gamma_{\varepsilon}}$ and $m \geq l$, we get, passing to the limit in (4.4), that the weak limit $z$ satisfies

$$
\left\{\begin{array}{l}
-z^{\prime \prime}-\frac{1}{m+r} z^{\prime}=e^{z} \quad \text { in }(-l,+\infty) \\
z(r) \leq 0, z(0)=z^{\prime}(0)=0 .
\end{array}\right.
$$

Then, setting $Z(s)=z(s-m)$ we derive that $Z$ satisfies

$$
\left\{\begin{array}{l}
-Z^{\prime \prime}-\frac{1}{r} Z^{\prime}=e^{Z} \quad \text { in }(m-l,+\infty) \\
Z(r) \leq 0, Z(m)=Z^{\prime}(m)=0
\end{array}\right.
$$

This Cauchy problem admits the unique solution (see [15])

$$
Z(s)=\log \frac{4 \alpha^{2} m^{\alpha+2} s^{\alpha-2}}{\left((\alpha+2) m^{\alpha}+(\alpha-2) s^{\alpha}\right)^{2}}
$$

where $\alpha=\sqrt{2 m^{2}+4}$. Let us show $m=l$. To this end, we can proceed as in Lemma 3.5 in [15]. For the sake of the completeness, we sketch it. We shall show that $z_{\varepsilon}\left(\left(r_{\varepsilon}-M_{\varepsilon}\right) / \gamma_{\varepsilon}\right) \rightarrow-\infty$ implies that $m=l$. Indeed, arguing as above, we have that for any $r \in\left[\left(r_{\varepsilon}-M_{\varepsilon}\right) / \gamma_{\varepsilon}, 0\right]$,

$$
z_{\varepsilon}^{\prime}(r)\left(\frac{M_{\varepsilon}}{\gamma_{\varepsilon}}+r\right) \leq-\left(\frac{M_{\varepsilon}}{\gamma_{\varepsilon}} r+\frac{1}{2} r^{2}\right) .
$$


If by contradiction we have that $m>l$, we deduce that $\frac{M_{\varepsilon}}{\gamma_{\varepsilon}}+r \geq m-l+o(1)$ where $o(1) \rightarrow 0$ as $\varepsilon \rightarrow 0$ and then we get that

$$
z_{\varepsilon}^{\prime}(r) \leq C \text { in }\left[\left(r_{\varepsilon}-M_{\varepsilon}\right) / \gamma_{\varepsilon}, 0\right]
$$

for a constant $C>0$ which is independent of small $\varepsilon>0$. On the other hand, by the mean value theorem, since $z_{\varepsilon}\left(\left(r_{\varepsilon}-M_{\varepsilon}\right) / \gamma_{\varepsilon}\right) \rightarrow-\infty$ and $z_{\varepsilon}(0)=0$ we deduce the existence of $\xi_{\varepsilon} \in\left(\frac{r_{\varepsilon}-M_{\varepsilon}}{\gamma_{\varepsilon}}, 0\right)$ such that $z_{\varepsilon}^{\prime}\left(\xi_{\varepsilon}\right) \rightarrow-\infty$ which gives a contradiction. So $m=l$. Now, from Lemmas 2.5, A.1 and the blow up procedure as above, we get

$$
\begin{aligned}
& 2=\lambda r_{i, \varepsilon}^{2} \int_{r_{\varepsilon}}^{1} v_{\varepsilon}^{2} e^{v_{\varepsilon}^{2}+v_{\varepsilon}^{1+\varepsilon}} r d r+o(1) \\
& =\frac{1}{2} \int_{\frac{r_{\varepsilon}-M_{\varepsilon}}{\gamma_{\varepsilon}}}^{\frac{1-M_{\varepsilon}}{\gamma_{\varepsilon}}}\left(\frac{z_{\varepsilon}(r)}{2 v_{\varepsilon}^{2}\left(M_{\varepsilon}\right)}+1\right)^{2} \times \\
& e^{z_{\varepsilon}(r)\left(\frac{z_{\varepsilon}(r)}{4 v_{\varepsilon}^{2}\left(M_{\varepsilon}\right)}+1\right)+v_{\varepsilon}^{1+\varepsilon}\left(M_{\varepsilon}\right)\left(\left|\frac{z_{\varepsilon}(r)}{2 v_{\varepsilon}^{2}\left(M_{\varepsilon}\right)}+1\right|^{1+\varepsilon}-1\right)}\left(\frac{M_{\varepsilon}}{\gamma_{\varepsilon}}+r\right) d r+o(1),
\end{aligned}
$$

where $o(1) \rightarrow 0$ as $\varepsilon \rightarrow 0$. Then using $m=l>0$ and Fatou's Lemma, we obtain

$$
2 \geq \frac{1}{2} \int_{0}^{\infty} e^{Z(s)} s d s=\sqrt{2 m^{2}+4}>2,
$$

a contradiction. This finishes Case 2 .

Case 3: $\frac{r_{\varepsilon}-M_{\varepsilon}}{\gamma_{\varepsilon}} \rightarrow 0$ occurs.

Repeating the procedure in Case 2 we can show $m=l=0$. As a consequence, we deduce

$$
z_{\varepsilon} \rightarrow z \text { in } C_{\mathrm{loc}}([0, \infty)) \cap C_{\mathrm{loc}}^{2}((0, \infty))
$$

and then, $z$ satisfies

$$
\left\{\begin{array}{l}
-z^{\prime \prime}-\frac{1}{r} z^{\prime}=e^{z} \quad \text { in }(0,+\infty) \\
z(r) \leq 0, z(0)=0
\end{array}\right.
$$

The previous equation can be integrate giving the solutions (see[15], p. 744$745)$

$$
z(r)=\log \left(\frac{4}{\delta^{2}} \frac{e^{\sqrt{2} \frac{\log r-y}{\delta}}}{\left(1+e^{\sqrt{2} \frac{\log r-y}{\delta}}\right)^{2}}\right)-2 \log r
$$

for some constants $\delta \neq 0, y \in \mathbb{R}$. Moreover a direct calculation shows

$$
z(r)=2 \log \frac{2}{\delta}-\frac{\sqrt{2}}{\delta} y+\left(\frac{\sqrt{2}}{\delta}-2\right) \log r-2 \log \left(1+e^{\sqrt{2} \frac{\log r-y}{\delta}}\right) .
$$

Since $z(0)=0$, we must have $\delta=1 / \sqrt{2}$. Then we clearly deduce $y=$ $\log 2 \sqrt{2}$. This completes the proof.

Proof of Proposition 4.1. The proposition follows from Lemmas 2.5, A.1 and 4.3 . 
Remark 4.4. If we consider a radial nodal solution $u_{p}$ to the problem

$$
\begin{cases}-\Delta u=|u|^{p-1} u & \text { in } B \\ u=0 & \text { on } \partial B,\end{cases}
$$

then in Proposition 3.1 of [15] it was proved that case 2 occurs for some suitable $m<0$. This shows that the shape of the nonlinearity plays a crucial role.

\section{BeHAVIOR OF $u_{\varepsilon}$ IN $B \backslash B_{r_{k, \varepsilon}}$}

Next we show the behavior on $B \backslash B_{r_{k, \varepsilon}}$. We set $u_{k+1, \varepsilon}:=\left.u_{\varepsilon}\right|_{B \backslash B_{r_{k, \varepsilon}}} \in$ $H_{0}^{1}(B)$ by zero extension. Then we have the following

Proposition 5.1. We get

$$
u_{k+1, \varepsilon} \rightarrow u_{0} \text { in } H_{0}^{1}(B),
$$

as $\varepsilon \rightarrow 0$ where $u_{0}$ is the least energy solution of (1.7).

First observe that we have already proved

$$
0<\lim _{\varepsilon \rightarrow 0} I_{\varepsilon}\left(u_{k+1, \varepsilon}\right)=\inf _{u \in \mathcal{N}_{0}} I_{0}(u)<2 \pi
$$

by Lemma 2.6. This means that the energy of $u_{k+1, \varepsilon}$ belongs to the suitable compactness region for Palais-Smale sequences [1. Although we do not ensure $\lim _{\varepsilon \rightarrow 0} I_{\varepsilon}^{\prime}\left(u_{k+1, \varepsilon}\right)=0$, we can accomplish the proof by the argument based Lions' concentration compactness result [19]. We refer the proof in [1] (and also [13]).

Proof of Proposition 5.1. Since $u_{\varepsilon}$ is bounded, we can assume, by choosing a sequence if necessary, that there exists a function $u_{0} \in H_{0}^{1}(\Omega)$ such that

$$
\begin{aligned}
& u_{\varepsilon} \rightarrow u_{0} \text { weakly in } H_{0}^{1}(B), \\
& u_{\varepsilon} \rightarrow u_{0} \text { in } L^{p}(B) \text { for all } p \geq 1, \\
& u_{\varepsilon} \rightarrow u_{0} \text { a.e. on } B
\end{aligned}
$$

as $\varepsilon \rightarrow 0$. Then, since $u_{i, \varepsilon} \rightarrow 0$ weakly in $H_{0}^{1}(B)$ for all $i=1,2, \cdots, k$, we also have

$$
\begin{aligned}
& u_{k+1, \varepsilon} \rightarrow u_{0} \text { weakly in } H_{0}^{1}(B), \\
& u_{k+1, \varepsilon} \rightarrow u_{0} \text { in } L^{p}(B) \text { for all } p \geq 1, \\
& u_{k+1, \varepsilon} \rightarrow u_{0} \text { a.e. on } B,
\end{aligned}
$$

as $\varepsilon \rightarrow 0$. Furthermore, since $\left\langle I_{\varepsilon}^{\prime}\left(u_{\varepsilon}\right), u_{\varepsilon}\right\rangle=0$, we get $\int_{B} f_{\varepsilon}\left(u_{\varepsilon}\right) u_{\varepsilon} d x$ is bounded. Then Lemma A.1 implies $f_{\varepsilon}\left(u_{\varepsilon}\right) \rightarrow f_{0}\left(u_{0}\right)$ in $L^{1}(B)$. We claim that $u_{0}$ is a nonnegative weak solution of (1.1) with $\varepsilon=0$. In fact, for all $\psi \in C_{0}^{\infty}(B)$, we get by the weak convergence of $u_{\varepsilon}$ and $L^{1}(B)$ convergence of $f_{\varepsilon}\left(u_{\varepsilon}\right)$,

$$
\begin{aligned}
0 & =\lim _{n \rightarrow \infty}\left\{\int_{B} \nabla u_{\varepsilon} \nabla \psi d x-\int_{B} f_{\varepsilon}\left(u_{\varepsilon}\right) \psi d x\right\} \\
& =\int_{B} \nabla u_{0} \nabla \psi d x-\int_{B} f_{0}\left(u_{0}\right) \psi d x .
\end{aligned}
$$


By a density argument we prove the claim. Next we shall show that there exists a constant $q>1$ such that

$$
\int_{B}\left|f_{\varepsilon}\left(u_{k+1, \varepsilon}\right)\right|^{q} d x \text { is bounded. }
$$

To see this, we observe that for a constant $\beta>1$, which will be determined later, there exists $C>0$ such that $\left|f_{\varepsilon}(t)\right| \leq C e^{\beta t^{2}}$ for all $t \in \mathbb{R}$ and small $\varepsilon>0$. Then for $q>1$, which will be also chosen later, we get

$$
\int_{B}\left|f_{\varepsilon}\left(u_{k+1, \varepsilon}\right)\right|^{q} d x \leq C \int_{B} e^{q \beta u_{k+1, \varepsilon}^{2}} d x=C \int_{B} e^{q \beta\left\|u_{k+1, \varepsilon}\right\|^{2} v_{\varepsilon}^{2}} d x
$$

where we set $v_{\varepsilon}:=u_{k+1, \varepsilon} /\left\|u_{k+1, \varepsilon}\right\|$. Notice $\left\|v_{\varepsilon}\right\|=1$ and $v_{\varepsilon} \rightarrow v_{0}$ weakly in $H_{0}^{1}(B)$ for a function $v_{0}$ with $0 \leq\left\|v_{0}\right\| \leq 1$. We claim that $v_{0} \neq 0$. If on the contrary $v_{0}=0$ we get $u_{0}=0$. Then Lemma A.1 shows $\int_{B} F_{\varepsilon}\left(u_{k+1, \varepsilon}\right) d x \rightarrow 0$ as $\varepsilon \rightarrow 0$. It follows that

$$
0<2 \lim _{\varepsilon \rightarrow 0} I_{\varepsilon}\left(u_{k+1, \varepsilon}\right)=\lim _{\varepsilon \rightarrow 0}\left\|u_{k+1, \varepsilon}\right\|^{2}<4 \pi .
$$

Consequently we can choose $\beta, q>1$ so that

$$
\int_{B}\left|f_{\varepsilon}\left(u_{k+1, \varepsilon}\right)\right|^{q} d x \leq C \int_{B} e^{q \beta\left\|u_{k+1, \varepsilon}\right\|^{2} v_{\varepsilon}^{2}} d x \leq C \int_{B} e^{4 \pi v_{\varepsilon}^{2}} d x
$$

for small $\varepsilon>0$. Notice that the Trudinger Moser inequality implies that the right hand side is bounded uniformly for small $\varepsilon>0$. Now setting $q^{\prime}>1$ so that $1 / q+1 / q^{\prime}=1$, we get by the Hölder inequality that

$$
\begin{aligned}
\left\|u_{k+1, \varepsilon}\right\|^{2} & =\int_{B} u_{k+1, \varepsilon} f_{\varepsilon}\left(u_{k+1, \varepsilon}\right) d x \\
& \leq\left(\int_{B}\left|u_{k+1, \varepsilon}\right|^{q^{\prime}} d x\right)^{\frac{1}{q^{\prime}}}\left(\int_{B}\left|f_{\varepsilon}\left(u_{k+1, \varepsilon}\right)\right|^{q} d x\right)^{\frac{1}{q}} \\
& \leq C\left(\int_{B}\left|u_{k+1, \varepsilon}\right|^{q^{\prime}} d x\right)^{\frac{1}{q^{\prime}}} .
\end{aligned}
$$

for a constant $C>0$ if $\varepsilon>0$ is small enough. Hence, we get $u_{k+1, \varepsilon} \rightarrow 0$ in $H_{0}^{1}(B)$ by (5.2). This contradicts (5.4). Therefore, we can assume $0<$ $\left\|v_{0}\right\|<1$. (If $\left\|v_{0}\right\|=1$, we finish the proof.) Then Lions' concentration compactness lemma (Theorem I.6 in [19]) proves

$$
\int_{B} e^{4 \pi p v_{\varepsilon}^{2}} d x \text { is bounded for all } p<\frac{1}{1-\left\|v_{0}\right\|^{2}} .
$$

Now recalling the facts that $\lim _{\varepsilon \rightarrow 0} I_{\varepsilon}\left(u_{k+1, \varepsilon}\right)<2 \pi, f_{0}(t) t-2 F_{0}(t) \geq 0$ for all $t \in \mathbb{R}$ and $\left\langle I_{0}^{\prime}\left(u_{0}\right), u_{0}\right\rangle=0$, we get a constant $\delta \in(0,1)$ such that

$$
\begin{aligned}
4 \pi(1-\delta) & =2 \lim _{\varepsilon \rightarrow 0} I_{\varepsilon}\left(u_{k+1, \varepsilon}\right) \\
& =\lim _{\varepsilon \rightarrow 0}\left\|u_{k+1, \varepsilon}\right\|^{2}-2 \int_{B} F_{0}\left(u_{0}\right) d x-\left\langle I_{0}^{\prime}\left(u_{0}\right), u_{0}\right\rangle \\
& \geq \lim _{\varepsilon \rightarrow 0}\left\|u_{k+1, \varepsilon}\right\|^{2}-\left\|u_{0}\right\|^{2} \\
& =\lim _{\varepsilon \rightarrow 0}\left\|u_{k+1, \varepsilon}\right\|^{2}\left(1-\left\|v_{0}\right\|^{2}\right) .
\end{aligned}
$$


This shows

$$
q \beta \lim _{\varepsilon \rightarrow 0}\left\|u_{k+1, \varepsilon}\right\|^{2} \leq \frac{4 \pi q \beta(1-\delta)}{1-\left\|v_{0}\right\|^{2}} .
$$

Put $p:=q \beta(1-\delta) /\left(1-\left\|v_{0}\right\|^{2}\right)$. Then, we can choose $\beta, q>1$ so that $p<1 /\left(1-\left\|v_{0}\right\|^{2}\right)$ and

$$
\int_{B}\left|f_{\varepsilon}\left(u_{k+1, \varepsilon}\right)\right|^{q} d x \leq C \int_{B} e^{4 \pi p v_{\varepsilon}^{2}} d x
$$

for small $\varepsilon>0$. Then (5.5) proves (5.3). Now choose $u_{0, \varepsilon} \in H_{0}^{1}(B)$ such that $u_{0, \varepsilon}=0$ on $\overline{B_{r_{k, \varepsilon}}}$ and $u_{0, \varepsilon} \rightarrow u_{0}$ in $H_{0}^{1}(B)$ as $\varepsilon \rightarrow 0$. (Define, for example, $u_{0, \varepsilon}:=\phi_{r_{k, \varepsilon}, 1} u_{0}$ where $\phi_{r_{k, \varepsilon}, 1}$ is a cut off function defined as in Section 2.) Then integration by parts gives that

$$
\begin{aligned}
\int_{B} \nabla u_{k+1, \varepsilon} \nabla\left(u_{k+1, \varepsilon}-u_{0, \varepsilon}\right) d x & =\int_{B \backslash B_{r_{k, \varepsilon}}}\left(-\Delta u_{k+1, \varepsilon}\right)\left(u_{k+1, \varepsilon}-u_{0, \varepsilon}\right) d x \\
& =\int_{B} f_{\varepsilon}\left(u_{k+1, \varepsilon}\right)\left(u_{k+1, \varepsilon}-u_{0, \varepsilon}\right) d x .
\end{aligned}
$$

Now again let $q^{\prime}>1$ be a constant such that $q^{-1}+q^{\prime-1}=1$. Then setting $o(1) \rightarrow 0$ as $\varepsilon \rightarrow 0$, and using the Hölder inequality, (5.3), and (5.2), we get

$$
\begin{aligned}
\left\|u_{k+1, \varepsilon}\right\|^{2}-\left\|u_{0}\right\|^{2} & =\int_{B} \nabla u_{k+1, \varepsilon} \nabla\left(u_{k+1, \varepsilon}-u_{0, \varepsilon}\right) d x+o(1) \\
& =\int_{B} f_{\varepsilon}\left(u_{k+1, \varepsilon}\right)\left(u_{k+1, \varepsilon}-u_{0, \varepsilon}\right) d x+o(1) \\
& \leq\left(\int_{B}\left|f_{\varepsilon}\left(u_{k+1, \varepsilon}\right)\right|^{q} d x\right)^{\frac{1}{q}}\left(\int_{B}\left|u_{k+1, \varepsilon}-u_{0, \varepsilon}\right|^{q^{\prime}} d x\right)^{\frac{1}{q^{\prime}}}+o(1) \\
& \rightarrow 0
\end{aligned}
$$

as $\varepsilon \rightarrow \infty$. Hence we get $u_{k+1, \varepsilon} \rightarrow u_{0}$ in $H_{0}^{1}(B)$ as $\varepsilon \rightarrow 0$. Finally, Lemma 2.6 proves that $u_{0}$ is the least energy solution of (1.7). This completes the proof.

Remark 5.2. From the result above, we get $\left\|u_{k+1, \varepsilon}\right\|_{L^{\infty}\left(\left(r_{k, \varepsilon}, 1\right)\right)}$ is bounded. To see this, observe that the strong convergence of $u_{k+1, \varepsilon}$ implies that for all $q>1, e^{u_{k+1, \varepsilon}^{2}}$ is bounded in $L^{q}(B)$ uniformly for small $\varepsilon>0$. Set $r_{k+1, \varepsilon}^{*} \in\left(r_{k, \varepsilon}, 1\right)$ so that $u_{r_{k+1}, \varepsilon}\left(r_{k+1, \varepsilon}^{*}\right)=\left\|u_{k+1, \varepsilon}\right\|_{L^{\infty}\left(\left(r_{k, \varepsilon}, 1\right)\right)}$. Then we get

$$
\begin{aligned}
\left|u_{k+1, \varepsilon}\left(r_{k+1, \varepsilon}^{*}\right)\right| & =\left|\int_{r_{k+1, \varepsilon}^{*}}^{1} f_{\varepsilon}\left(u_{k+1, \varepsilon}\right) r \log r d r\right| \\
& \leq\left(\int_{r_{k+1, \varepsilon}^{*}}^{1} f_{\varepsilon}\left(u_{k+1, \varepsilon}\right)^{2} r d r\right)^{\frac{1}{2}}\left(\int_{r_{k+1, \varepsilon}^{*}}^{1} r \log ^{2} r d r\right)^{\frac{1}{2}} \\
& \leq C
\end{aligned}
$$

for a constant $C>0$ if $\varepsilon>0$ is sufficiently small. This proves the claim.

Remark 5.3. The previous remark shows

$$
\lim _{\varepsilon \rightarrow 0} r_{k, \varepsilon} u_{\varepsilon}^{\prime}\left(r_{k, \varepsilon}\right)=0 .
$$


To show this, set $r_{k+1, \varepsilon}^{*} \in\left(r_{k, \varepsilon}, 1\right)$ as above. First observe that $r_{k+1, \varepsilon}^{*} \rightarrow 0$ as $\varepsilon \rightarrow 0$. If not, we have a constant $r_{0} \in(0,1)$ such that $r_{k+1, \varepsilon}^{*} \rightarrow r_{0}$ as $\varepsilon \rightarrow 0$ by choosing a sequence if necessary. Then since $u_{k+1, \varepsilon} \rightarrow u_{0}$ a.e. on $B$, we have $u_{0}(r) \leq u_{0}\left(r_{0}\right)$ for a.a. $r \in\left(0, r_{0}\right)$. But, since $u_{0}$ is a positive radial solution of (1.7), the result in [14] shows $u^{\prime}(r)<0$ for all $r \in(0,1)$. This is a contradiction. Finally, integrating (1.9) over $\left(r_{k, \varepsilon}, r_{k+1, \varepsilon}^{*}\right)$, we get by the previous remark that

$$
\left|r_{k, \varepsilon} u_{\varepsilon}^{\prime}\left(r_{k, \varepsilon}\right)\right|=\left|\int_{r_{k, \varepsilon}}^{r_{k+1, \varepsilon}^{*}} f_{\varepsilon}\left(u_{\varepsilon}\right) r d r\right| \leq C r_{k+1, \varepsilon}^{*} .
$$

for some constant $C>0$. This completes the proof.

\section{Proof of MAIN THEOREMS}

We finally conclude the proof of our main theorems.

Proof of Theorem 1.2. The proof of (1.11) is given in Lemma 2.4, (1.12) is shown in Corollary [3.2, Proposition 4.1 and Proposition 5.1, (1.13) is shown in Corollary 3.2. Proposition 4.1 and Proposition 5.1. So we have only to show (1.10), i.e.

$$
u_{\varepsilon} \rightarrow(-1)^{k} u_{0} \text { in } C_{\mathrm{loc}}^{2}((0,1])
$$

as $\varepsilon \rightarrow 0$. To prove this, we may assume that $u_{\varepsilon}$ satisfies

$$
\left\{\begin{array}{l}
-u_{\varepsilon}^{\prime \prime}-\frac{1}{r} u_{\varepsilon}^{\prime}=\lambda u_{\varepsilon} e^{u_{\varepsilon}^{2}+u_{\varepsilon}^{1+\varepsilon}}, u_{\varepsilon}>0 \text { in }\left(r_{k, \varepsilon}, 1\right), \\
u_{\varepsilon}\left(r_{k, \varepsilon}\right)=u_{\varepsilon}(1)=0 .
\end{array}\right.
$$

Now choose any compact subset $K \subset \subset(0,1]$. For all $r \in K$, we may suppose $r_{k, \varepsilon}<r$ by Lemma 2.4. Then multiplying (6.1) by $r$ and integrating over $\left(r_{k, \varepsilon}, r\right)$ we have

$$
r u_{\varepsilon}^{\prime}(r)=r_{k, \varepsilon} u_{\varepsilon}^{\prime}\left(r_{k, \varepsilon}\right)-\int_{r_{k, \varepsilon}}^{r} f_{\varepsilon}\left(u_{\varepsilon}\right) r d r .
$$

Then Remark 5.3 and Lemma A.1 prove that $r u_{\varepsilon}^{\prime}$ is bounded uniformly on $K$ for small $\varepsilon$. In particular, $u_{\varepsilon}^{\prime}$ is bounded uniformly on $K$ for small $\varepsilon$. Furthermore, since for any $r \in K$, we have

$$
u_{\varepsilon}(r)=-\int_{r}^{1} u_{\varepsilon}^{\prime}(s) d s,
$$

we derive $\left\|u_{\varepsilon}\right\|_{C^{1}(K)}$ is bounded uniformly for small $\varepsilon$. Then the ArzelaAscoli theorem ensures $u_{\varepsilon} \rightarrow u_{0}$ uniformly on $K$ as $\varepsilon \rightarrow 0$. Finally, using (6.1), we show $u_{\varepsilon} \rightarrow u_{0}$ in $C^{2}(K)$ as $\varepsilon \rightarrow 0$. This finishes the proof.

Proof of Theorem 1.3. For $i=0$ the proof is given in Proposition 3.1. The case $i=1, . ., k$ is considered in Proposition 4.1.

Using the blow up results above, we get the following remark.

Remark 6.1. We have

$$
\lim _{\varepsilon \rightarrow 0} \frac{\left\|u_{\varepsilon}\right\|_{L^{\infty}\left(\left(r_{i, \varepsilon}, r_{i+1, \varepsilon}\right)\right)}}{\left\|u_{\varepsilon}\right\|_{L^{\infty}\left(\left(r_{i-1, \varepsilon}, r_{i, \varepsilon}\right)\right)}}=0
$$


for all $i=1,2, \cdots, k$. Let us show the proof. For $i=k$, the proof is obvious by Corollary 3.2, Lemma 4.2 and Remark 5.2. Then for $i=1,2, \cdots, k-1$, set $M_{i, \varepsilon} \in[0,1)$ so that $\left|u_{\varepsilon}\left(M_{i, \varepsilon} r_{i, \varepsilon}\right)\right|=\left\|u_{\varepsilon}\right\|_{L^{\infty}\left(\left(r_{i-1, \varepsilon}, r_{i, \varepsilon}\right)\right)}, r_{i, \varepsilon}^{*}:=M_{i, \varepsilon} r_{i, \varepsilon}$ and $\gamma_{i, \varepsilon}=2 u_{\varepsilon}\left(r_{i, \varepsilon}^{*}\right) f_{\varepsilon}\left(u_{\varepsilon}\left(r_{i, \varepsilon}^{*}\right)\right)>0$. Then integrating (1.9) over $\left(r_{i, \varepsilon}^{*}, r_{i+1, \varepsilon}^{*}\right)$ shows

$$
\int_{r_{i, \varepsilon}^{*}}^{r_{i, \varepsilon}} f_{\varepsilon}\left(u_{\varepsilon}\right) r d r=-\int_{r_{i, \varepsilon}}^{r_{i+1, \varepsilon}^{*}} f_{\varepsilon}\left(u_{\varepsilon}\right) r d r .
$$

Hence putting $v_{i, \varepsilon}(r)=\left|u_{i, \varepsilon}\left(r_{i, \varepsilon} r\right)\right|, v_{i+1, \varepsilon}(r)=\left|u_{i+1, \varepsilon}\left(r_{i+1, \varepsilon} r\right)\right|$ and $z_{j, \varepsilon}(r)=$ $\left.2\left|u_{\varepsilon}\left(r_{j, \varepsilon}^{*}\right)\right|\left(\left|v_{j, \varepsilon}\left(\gamma_{j, \varepsilon} r+M_{j, \varepsilon}\right)\right|-\mid u_{\varepsilon}\left(r_{j, \varepsilon}^{*}\right)\right) \mid\right)$ for $j=i, i+1$, we get by the blow up procedure as above,

$$
\begin{aligned}
& \frac{1}{2\left|u_{\varepsilon}\left(r_{i, \varepsilon}^{*}\right)\right|} \times \\
& \int_{0}^{\frac{1-M_{i, \varepsilon}}{\gamma_{i, \varepsilon}}}\left(\frac{z_{i, \varepsilon}(r)}{2 v_{i, \varepsilon}^{2}\left(M_{i, \varepsilon}\right)}+1\right) \times
\end{aligned}
$$

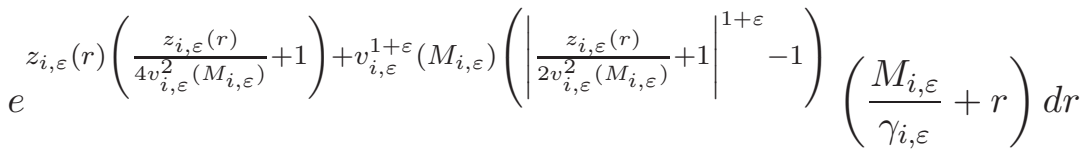

$$
\begin{aligned}
& =\frac{1}{2\left|u_{\varepsilon}\left(r_{i+1, \varepsilon}^{*}\right)\right|} \times \\
& \int_{\frac{r_{i, \varepsilon}}{r_{i+1, \varepsilon}}-M_{i+1, \varepsilon}}^{\gamma_{i+1, \varepsilon}}\left(\frac{z_{i+1, \varepsilon}(r)}{2 v_{i+1, \varepsilon}^{2}\left(M_{i+1, \varepsilon}\right)}+1\right) \times \\
& e^{z_{i+1, \varepsilon}(r)\left(\frac{z_{i+1, \varepsilon}(r)}{4 v_{i+1, \varepsilon}^{2}\left(M_{\varepsilon}\right)}+1\right)+v_{i+1, \varepsilon}^{1+\varepsilon}\left(M_{i+1, \varepsilon}\right)}\left(\left|\frac{z_{i+1, \varepsilon}(r)}{2 v_{i+1, \varepsilon}^{2}\left(M_{i+1, \varepsilon}\right)}+1\right|^{1+\varepsilon}-1\right)\left(\frac{M_{i+1, \varepsilon}}{\gamma_{i+1, \varepsilon}}+r\right) d r \\
& =o\left(\frac{1}{2\left|u_{\varepsilon}\left(r_{i+1, \varepsilon}^{*}\right)\right|}\right)
\end{aligned}
$$

as $\varepsilon \rightarrow 0$ since $\lim _{\varepsilon \rightarrow 0} \frac{r_{i, \varepsilon} / r_{i+1, \varepsilon}-M_{i+1, \varepsilon}}{\gamma_{i+1, \varepsilon}}=0$. Finally using our blow up results and the Fatou lemma for the integral on the left hand side, we get the desired conclusion.

\section{Appendix A. Some BASIC FaCts}

In the following, let $\left(\varepsilon_{n}\right) \subset \mathbb{R}^{+}$be any sequence such that $\varepsilon_{n} \rightarrow 0$ as $n \rightarrow \infty$.

Lemma A.1. Let $\left(u_{n}\right) \subset H_{0}^{1}(B)$ be a bounded sequence such that $u_{n} \rightarrow u$ weakly in $H_{0}^{1}(B)$ and $u_{n} \rightarrow u$ a.e. on $B$ as $n \rightarrow \infty$ for a function $u$. Furthermore, assume

$$
\sup _{n} \int_{B} f_{\varepsilon_{n}}\left(u_{n}\right) u_{n} d x<\infty .
$$

Then we have

$$
\lim _{n \rightarrow \infty} \int_{B} f_{\varepsilon_{n}}\left(\left|u_{n}\right|\right) d x=\int_{B} f_{0}(|u|) d x
$$


and

$$
\lim _{n \rightarrow \infty} \int_{B} F_{\varepsilon_{n}}\left(u_{n}\right) d x=\int_{B} F_{0}(u) d x .
$$

Proof. Similar to the proof of 4) of Lemma 3.1 in [2].

Lemma A.2. We have

$$
\liminf _{\varepsilon \rightarrow 0} \inf _{u \in \mathcal{N}_{\varepsilon}} I_{\varepsilon}(u)>0 .
$$

Proof. If not, we have sequences $\left(\varepsilon_{n}\right) \subset \mathbb{R}^{+}$and $\left(u_{n}\right) \subset \mathcal{N}_{\varepsilon_{n}}$ such that $\lim _{n \rightarrow \infty} I_{\varepsilon_{n}}\left(u_{n}\right)=0$. Then since $\lambda<\lambda_{1}$, analogously with Step 1 in the proof of Lemma 3.4 in [2], we can get a contradiction. This proves the lemma.

Lemma A.3. Let $\left(\mu_{n}\right) \subset \mathbb{R}^{+}$and $\left(u_{n}\right) \subset H_{0}^{1}(B)$ be sequences such that $\mu_{n} \leq 1$ for all $n$ and further,

$$
\begin{aligned}
& J_{n}\left(u_{n}\right):=\int_{B}\left|\nabla u_{n}\right|^{2} d x-\mu_{n} \int_{B} F_{\varepsilon_{n}}\left(u_{n}\right) d x \rightarrow c \in(0,2 \pi) \text { and } \\
& J_{n}^{\prime}\left(u_{n}\right) \rightarrow 0 \text { in } H^{-1}(B),
\end{aligned}
$$

as $n \rightarrow \infty$. Then $u_{n} \rightarrow u$ in $H_{0}^{1}(B)$ up to a subsequence.

Proof. Similar to 1) on p404 in [2].

\section{REFERENCES}

[1] Adimurthi, Positive solutions of the semilinear Dirichlet problem with critical growth in the unit disc in $\mathbb{R}^{2}$, Proc. Indian Acad. Sci., Mathematical Sciences 99 (1989) 4973.

[2] Adimurthi, Existence of positive solutions of the semilinear Dirichlet problem with critical growth for the $n$-Laplacian, Ann. Scuola Norm. Sup. Pisa Cl. Sci. (4) 17 (1990) 393-413.

[3] Adimurthi and O. Druet, Blow-up analysis in dimension 2 and a sharp form of Trudinger-Moser inequality, Comm. in PDE. 29 (2004) 295-322.

[4] Adimurthi and M. Struwe, Global compactness properties of semilinear elliptic equations with critical exponential growth, J. Funct. Anal. 175 (2000) 125167.

[5] Adimurthi and Yadava, Nonexistence of Nodal Solutions of Elliptic Equations with Critical Growth in $\mathbb{R}^{2}$, Trans. Amer. Math. Soc. 332 (1992) 449458.

[6] Adimurthi and S.L. Yadava, Multiplicity results for semilinear elliptic equations in a bounded domain of $\mathbb{R}^{2}$ involving critical exponents. Ann. Scuola Norm. Sup. Pisa Cl. Sci. (4) 17 (1990) 481504.

[7] Adimurthi and S. Prashanth, Failure of Palais-Smale condition and blow-up analysis for the critical exponent problem in $\mathbb{R}^{2}$, Proc. Indian Acad. Sci. Math. Sci. 107 (1997) 283317.

[8] F.V. Atkinson, H. Brezis and L.A. Peletier, Nodal solutions of elliptic equations with critical Sobolev exponents. J. Diff. Eqns 85 (1990), 151170.

[9] F.V. Atkinson and L.A. Peletier, Ground states and Dirichlet problems for $-\Delta u=f(u)$ in $\mathbb{R}^{2}$. Arch. Rational Mech. Anal. 96 (1986) 103-127.

[10] M. Ben Ayed, K. El Mehdi and F. Pacella, Classification of low energy sign-changing solutions of an almost critical problem, Jour. Funct. Anal. 250 (2007) 347-373

[11] L. Carleson, A. Chang, On the existence of an extremal fun ction for an inequality of J. Moser, Bull. Sci. Math. 110 (1986) 113-127.

[12] F. De Marchis, I. Ianni and F. Pacella, A Morse index formula for radial solutions of Lane-Emden problems, preprint arXiv:1605.03357.

[13] D.G. de Figueiredo, O.H. Miyagaki and B. Ruf, Elliptic equations in $\mathbb{R}^{2}$ with nonlinearities in the critical growth range, Calc. Var. 3 (1995) 139-153. 
[14] B. Gidas, W.M. Ni and L. Nirenberg, Symmetry and related properties via the maximum principle, Comm. Math. Phys. 68 (1979) 209-243.

[15] M. Grossi, C. Grumiau and F. Pacella, Lane Emden problems with large exponents and singular Liouville equations, Jour. Math. Pures Appl., (2014), 101, 735-754.

[16] M. Grossi and A. Pistoia, Multiple blow-up phenomena for the sinh-Poisson equation", Arc. Rat. Mech. Anal., (2013), 209, 287-320.

[17] A. Iacopetti and F. Pacella, Asymptotic analysis for radial sign-changing solutions of the Brezis-Nirenberg problem in low dimensions. Contributions to nonlinear elliptic equations and systems, 325343, Progr. Nonlinear Differential Equations Appl., 86, Birkhuser/Springer, Cham, 2015.

[18] A. Iacopetti and G. Vaira, Sign-changing blowing-up solutions for the BrezisNirenberg problem in dimensions four and five, Ann. Scuola Norm. Sup. Pisa, to appear.

[19] P. L. Lions, The concentration-compactness principle in the calculus of variations. The limit case. Part 1., Rev. Mat. Iberoamericana, 1 (1985), 145-201.

[20] J.K. Moser , A sharp form of an inequality by N. Trudinger, Indiana Univ. Math. J., 20, (1971) 1077-1092.

[21] T. Ogawa and T. Suzuki, Nonlinear elliptic equations with critical growth related to the Trudinger inequality, Asym. Anal. 12 (1996) 25-40.

[22] T. Ogawa and T. Suzuki, Two-dimensional elliptic equation with critical nonlinear growth, Trans. Amer. Math. Soc. 350 (1998) 4897-4918.

[23] S. I. Pohozaev, The Sobolev embedding in the case $p l=n$, Proc. of the Technical Scientific Conference on Advances of Scientific Research 1964-1965, Mathematics Section, (Moskov. Energet. Inst., Moscow), (1965), 158-170.

[24] A. Pistoia, T. Weth, Sign changing bubble tower solutions in a slightly subcritical semilinear Dirichlet problem, Ann. Inst. H. Poincaré Anal. Non Lin. 24 (2007) 325340.

[25] A. Strauss, Existence of solitary waves in higher dimensions, Comm. Math. Phys. 55 (1977) 149-162.

[26] N.S. Trudinger, On imbeddings into Orlicz spaces and some applications, J. Math. Mech., 17, 473-483.

† Dipartimento di Matematica, Università di Roma "La SAPienza", massimo.grossi@uniroma1. it $\ddagger$ Muroran Institute of TeChnology, naimen@mmm. muroran-it.ac. JP 